\title{
Die Lissabon-Strategie der Europäischen Union: eine kritische Bewertung
}

\section{Einleitung}

Im Frühjahr des vergangenen Jahres legte die Europäische Kommission unter dem Titel „Europa 2020“ eine „Wachstumsstrategie für das kommende Jahrzehnt“" vor. ${ }^{1}$ Die dem zugrunde liegende Idee ist nicht neu, gab sich die Europäische Union (EU) doch bereits vor zehn Jahren einen ähnlichen wirtschafts- und arbeitsmarktpolitischen Rahmen, namentlich die „Lissabon-Strategie““. Während die Einrichtungen der EU bereits dazu übergegangen sind, die Vorzüge des neuen Planwerks zu würdigen, soll an dieser Stelle der Blick zunächst in die Vergangenheit gerichtet werden, geht es mithin v.a. um die bisher erkennbaren Ergebnisse einer solchen unionsweiten „Wachstumsstrategie“.

Zunächst ist festzustellen, dass beide Planwerke vor dem Hintergrund der Finanz-, Wirtschafts- und Staatsschuldenkrise zwar an politischer Aufmerksamkeit verloren haben, die darin skizzierten Problemstellungen und Aufgabenfelder sich jedoch nach wie vor als aktuell erweisen: Weiterentwicklung des Binnenmarktes, Stärkung der Wettbewerbsfähigkeit, Förderung von Innovation und Bildung, Intensivierung des Umweltschutzes, zukunftsfähige Ausrichtung der Energiepolitik, Modernisierung der sozialen Sicherungssysteme, Sanierung der Haushalte und Koordinierung der Wirtschaftspolitik (nicht nur) im Euroraum. Die meisten der bislang vorliegenden Evaluationen zur „Lissabon-Strategie“ befassen sich indessen mit der Frage nach dem Sinn eines zehnjährigen Planungszeitraums und beurteilen den Erfolg des Planwerks in einigen ausgewählten Bereichen, erbringen aber nur in Teilen eine empirisch-analytisch dringend angezeigte, gesamthaft ausgerichtete Bestandsaufnahme unter Einbezug der Koordinationsleistungen sowie - vor allem - des Vollzugs und seiner Ergebnisse. ${ }^{3}$ Der folgende Beitrag sucht dieses Defizit auszugleichen.

1 Europäische Kommission: Europa 2020. Eine Strategie für intelligentes, nachhaltiges und integratives Wachstum, KOM(2010) 2020.

2 Europäischer Rat: Schlussfolgerungen des Vorsitzes, Lissabon, 23. und 24.03.2000.

3 Vgl. etwa Begg, I.: Lisbon II, Two Years on: An Assessment of the Partnership for Growth and Jobs Special CEPS Report. Centre for European Policy Studies, 2007; Bongardt, A./Torres, F.: Institutions, 


\section{Strategische Grundausrichtung}

Die strategische Ausrichtung der „Lissabon-Strategie“ fußte auf der Wahrnehmung eines (aktuellen wie absehbaren) ökonomischen Wettbewerbsdrucks im Rahmen einer sich rapide internationalisierenden Welt. Überkommene Modernisierungsansätze und selbst der ungewöhnlich starke Wirtschaftsaufschwung der sog. New Economy zu Beginn des vergangenen Jahrzehnts konnten den eklatanten Abstand bei Produktivität und Wettbewerbsfähigkeit zwischen der EU auf der einen und den USA und Japan auf der anderen Seite kaum ausgleichen; das kontinuierlich hohe Wachstum in den Schwellenländern wurde und wird als zusätzliche Herausforderung begriffen. Die Auswirkungen der Globalisierung sah man als „Quantensprung“, der ,,jeden Aspekt des Alltags“ berühre, während eine Kombination aus hoher struktureller Arbeitslosigkeit und niedrigen Beschäftigungsquoten als Gefahr für die Funktionsfähigkeit der sozialen Sicherungssysteme galt. ${ }^{4}$ Nach der formalen Schaffung des Europäischen Binnenmarktes 1993 und der Einführung des Euro 1999 bedurfte die EU zudem eines neuen wirtschaftspolitischen Großprojekts, um ihre Eigenlegitimation jenseits der territorialen Erweiterung durch eine verbesserte Koordination der verfolgten Einzelmaßnahmen zu bestätigen.

Die Grundidee bestand in der Stärkung der Wettbewerbsfähigkeit im Kontext ökologischer Nachhaltigkeit und sozialer Integration. Der Europäische Rat beschloss daher,

„die Union zum wettbewerbsfähigsten und dynamischsten wissensbasierten Wirtschaftsraum der Welt zu machen - einem Wirtschaftsraum, der fähig ist, ein dauerhaftes Wirtschaftswachstum mit mehr und besseren Arbeitsplätzen und einem größeren sozialen Zusammenhalt zu erzielen. “5

Governance and Economic Growth in the EU: Is There a Role for the Lisbon Strategy?, in: Intereconomics January/February 2007, 32 - 42; Collignon, St.: Vorwärts mit Europa - Für eine demokratische und progressive Reform der Lissabon-Strategie. Friedrich-Ebert-Stiftung, 2008; Institute for Strategic and International Studies (Hg.): The Lisbon Agenda and European Governance Workshop IV, 29 February 2008, Technical Report, London, 2008.

4 Diese und die folgenden Ausführungen entsprechen der Analyse des Europäischen Rates: Schlussfolgerungen des Vorsitzes, Lissabon, 23. und 24.03.2000.

5 Ebd., Rn. 5. 


\section{Ziele und geplante Umsetzung}

Grundsätzlich verfolgte die Lissabon-Strategie also zwei übergreifende Ziele, die hinsichtlich der einzubeziehenden Politikfelder wie folgt ausdifferenziert wurden:

- Stärkung der Wettbewerbsfähigkeit: Wirtschafts- und Strukturreformen, Innovationsförderung, Konzertierung bildungspolitischer Maßnahmen, Anpassung an die Wissensgesellschaft, Weiterentwicklung des EU-Binnenmarktes, Verbesserung der makroökonomischen Politikkoordination und der Rahmenbedingungen für kleine und mittelständische Unternehmen (KMU).

- Sicherung des sozialen Zusammenhalts: Steigerung der Beschäftigtenzahl, Vermeidung sozialer Ausgrenzung, Förderung von Bildung und Ausbildung, Anpassung der sozialen Sicherungssysteme an die Auswirkungen der demographischen Entwicklung. ${ }^{6}$

Die Umsetzung dieser Ziele sollte auf allen gebietskörperschaftlichen Ebenen und unter Einbezug aller bedeutenden Akteure, Instrumente und Finanzierungsquellen erfolgen. Vorausgesetzt wurden eine engere Zusammenarbeit der Mitgliedstaaten untereinander sowie eine verstärkte Anbindung an die Organe der Union im Allgemeinen und die Europäischen Kommission im Besonderen. Zudem beschloss man den Einbezug zahlreicher Nichtregierungsorganisationen, der Sozialverbände und Tarifparteien, des Unternehmenbereichs und der Forschungseinrichtungen. Als zentrales Instrument sollte die sog. Offene Methode der Koordinierung (OMK) dienen, die auf der Grundlage der Prinzipien des Erfahrungsaustauschs, des gegenseitigen Vergleichs (anhand konkreter benchmarks) und der Erstellung gemeinsamer Leitlinien die nationalstaatliche Politikformulierung europaweit zu bündeln sucht.

Mit der OMK zielte man auf jene Politikbereiche, die zwar ganz oder in Teilen im Kompetenzbereich der Mitgliedstaaten verblieben, sich für die Umsetzung der beschlossenen Zielsetzungen jedoch als bedeutsam erwiesen. Der Europäische Rat übernahm in diesem Kontext die Festlegung der inhaltlichen Vorgaben und bemühte sich somit, einer umfassenden Koordinationsfunktion zu entsprechen, während die Mitgliedstaaten Reformprogramme entwarfen, die seitens der Kommission durch jährliche Berichte sukzessiv überwacht und, ergänzt um einen Empfehlungskatalog, kommentiert werden sollten. Zur „Messung“ der Umsetzungsergebnisse sah man zahlreiche Indikatoren vor; ${ }^{7}$ die Finanzierung

6 Ebd., Rn. 8-34.

7 Siehe hierzu die Übersicht von Eurostat: http://epp.eurostat.ec.europa.eu unter „Structural Indicators“. 
der Einzelmaßnahmen schließlich sollte durch die Union, unterschiedliche EUFörderfonds, die Europäische Investitionsbank (EIB), die Mitgliedstaaten und private Einrichtungen bzw. Unternehmen gewährleistet werden. Zeitgeistgeprägt standen dabei Public-Private-Partnerships im Zentrum der Überlegungen.

Die große Erweiterungsrunde des Jahres 2004, der Wechsel im Amt des Kommissionspräsidenten und eine erste (ernüchternde) Zwischenbilanz gaben im Jahr 2005 dann Anlass zu einer Neuausrichtung der „Lissabon-Strategie“. 8 Sämtliche Maßnahmen sollten sich künftig an zwei Zielen orientieren: Wachstum und Beschäftigung. Zwar wurde die Strategie erstmals auch um eine Reihe umweltpolitischer Zielsetzungen ergänzt, ${ }^{9}$ allerdings mussten sich diese - wie auch der gesamte Bereich Soziales - den beiden benannten Kernzielen unterordnen. Der Schwerpunkt wurde auf die Förderung von Forschung und Innovation, die Weiterentwicklung der Informations- und Wissensgesellschaft sowie die Unterstützung kleiner und mittelständischer Unternehmen (KMU) verlagert, die europäische Kohäsionspolitik zudem explizit als Teil des Planwerks definiert. Diese vermeintlich umfassende inhaltliche Umorientierung führte faktisch jedoch nur zu geringfügigen Modifikationen im Katalog der bereits $a b$ ovo vorgesehenen Einzelmaßnahmen; statt von einer „Neuausrichtung“ wäre also eher von einer Schwerpunktverlagerung zu sprechen.

Schließlich vereinfachte man das Umsetzungs- und Evaluationsverfahren und wurde der Einjahres- durch einen Dreijahreszyklus ersetzt. Auf Grundlage der Fortschrittsberichte der Kommission verabschiedete der Europäische Rat fortan „Integrierte Leitlinien“ und bündelte darin die wirtschafts- und beschäftigungspolitischen Vorgaben. Die Mitgliedstaaten erarbeiteten auf dieser Grundlage „Nationale Aktionspläne“, ${ }^{10}$ während die Kommission für ihren Zuständigkeitsbereich ein dreijähriges „Lissabon-Programm der Gemeinschaft“ entwarf. Nach Ablauf des Dreijahreszeitraums sollten Leitlinien und Aktionspläne der Mitgliedstaaten wie der Gemeinschaft geprüft und ggf. angepasst werden; die Kommission legte darüber hinaus wie bislang einen Jahresbericht vor.

Die nachfolgende, nach Politikfeldern gegliederte Übersicht (Tab. 1) summiert die materiellen Zielsetzungen der „Lissabon-Strategie“ unter Berücksichtigung der zur Zielerreichung vorgesehenen Einzelmaßnahmen.

8 Die folgenden Ausführungen beziehen sich auf: Europäischer Rat: Schlussfolgerungen des Vorsitzes, Brüssel, 22. und 23.03.2005.

9 Europäische Kommission: KOM (2001) 31.

10 Europäischer Rat: a.a.O., 2005, 8f. 
Tabelle 1: Zielsetzungen und Vollzugsplanung der Lissabon-Strategie (2005)

\begin{tabular}{|c|c|}
\hline Ziel & Vorgesehene Maßnahmen zur Umsetzung \\
\hline \multicolumn{2}{|c|}{ Wettbewerbsfähigkeit - Informationsgesellschaft } \\
\hline $\begin{array}{l}\text { Verbesserte Koordination } \\
\text { der IKT-Politik }\end{array}$ & $\begin{array}{l}\text { Erstellung eines „eEurope“-Aktionsplans, Nutzung der } \\
\text { Offenen Methode der Koordinierung (OMK) }\end{array}$ \\
\hline $\begin{array}{l}\text { Liberalisierung des } \\
\text { Informationssektors }\end{array}$ & $\begin{array}{l}\text { Harmonisierung des rechtlichen Rahmens, } \\
\text { Ausbau des Frequenzangebots für mobile Dienste }\end{array}$ \\
\hline $\begin{array}{l}\text { Vermittlung grundlegender } \\
\text { IKT-Fähigkeiten }\end{array}$ & Internetzugang für alle Schulen in der EU \\
\hline $\begin{array}{l}\text { Steigerung der Internetnutzung der } \\
\text { öffentlichen Verwaltung }\end{array}$ & Einrichtung von Internetportalen, Schulungen \\
\hline $\begin{array}{l}\text { Ausbau des elektronischen } \\
\text { Geschäftsverkehrs }\end{array}$ & $\begin{array}{l}\text { Schaffung eines einheitlichen Rechtsrahmens, } \\
\text { Förderung der Nutzung durch Unternehmen und Bürger }\end{array}$ \\
\hline $\begin{array}{l}\text { Ausbau schneller } \\
\text { Internetverbindungen }\end{array}$ & $\begin{array}{l}\text { Spezifische Fördermaßnahmen für strukturschwache und } \\
\text { dünn besiedelte Gebiete, Rückgriff auf Mittel der } \\
\text { Europäischen Investitionsbank (EIB) }\end{array}$ \\
\hline \multicolumn{2}{|c|}{ Wettbewerbsfähigkeit - Europäischer Forschungsraum } \\
\hline Verbesserter Informationsfluss & Einführung eines „Europäischen Innovationsanzeigers“ \\
\hline $\begin{array}{l}\text { Effektive Nutzung der Mittel für } \\
\text { Forschung und Entwicklung (FuE) }\end{array}$ & $\begin{array}{l}\text { Erhöhung der Ausgaben für FuE auf } 3 \% \text { des BIP, Ver- } \\
\text { netzung von Forschungsprogrammen, Nutzung der OMK }\end{array}$ \\
\hline $\begin{array}{l}\text { Verbesserung der Ausgangs- } \\
\text { bedingungen für FuE }\end{array}$ & $\begin{array}{l}\text { Anpassung steuerpolitischer Anreize, Förderung von } \\
\text { Risikokapital-Investitionen, Rückgriff auf EIB-Mittel }\end{array}$ \\
\hline $\begin{array}{l}\text { Europäisches wissenschaftliches } \\
\text { Kommunikationsnetz }\end{array}$ & $\begin{array}{l}\text { Schaffung und Betreuung der notwendigen Infrastrukturen, } \\
\text { Rückgriff auf EIB-Mittel }\end{array}$ \\
\hline Abbau von Mobilitätshindernissen & $\begin{array}{l}\text { Vereinfachte Anerkennung von Leistungsnachweisen, } \\
\text { finanzielle Förderung wissenschaftlicher Mobilität }\end{array}$ \\
\hline Gemeinschaftspatent & Schaffung eines einheitlichen Rechtsrahmens \\
\hline \multicolumn{2}{|c|}{ Wettbewerbsfähigkeit-Standortbedingungen } \\
\hline Bürokratieabbau & $\begin{array}{l}\text { Erstellung einer „Europäischen Charta für kleine } \\
\text { Unternehmen“, Nutzung der OMK }\end{array}$ \\
\hline $\begin{array}{l}\text { Ausbau der Schnittstellen } \\
\text { zwischen Ausbildung, Wissenschaft } \\
\text { und Praxis }\end{array}$ & $\begin{array}{l}\text { Förderung durch die EIB und den Europäischen } \\
\text { Investitionsfonds (EIF) }\end{array}$ \\
\hline \multicolumn{2}{|c|}{ Wettbewerbsfähigkeit-Binnenmarkt } \\
\hline $\begin{array}{l}\text { Ständige Verbesserung } \\
\text { des Binnenmarkts }\end{array}$ & $\begin{array}{l}\text { Liberalisierung des Dienstleistungssektors, des Gas- und } \\
\text { Strommarkts, der Postdienste und des Transportsektors }\end{array}$ \\
\hline Überprüfung der Beihilferegeln & $\begin{array}{l}\text { Abbau von Subventionen, Ausrichtung auf die „Lissabon- } \\
\text { ziele“, elektronische Abwicklung öffentlicher Aufträge }\end{array}$ \\
\hline \multicolumn{2}{|c|}{ Wettbewerbsfähigkeit - Integration der Finanzmärkte } \\
\hline $\begin{array}{l}\text { Weiterentwicklung des Binnen- } \\
\text { marktes für Finanzdienstleistungen }\end{array}$ & $\begin{array}{l}\text { Entwicklung und Einführung eines gemeinsamen } \\
\text { Rechtsrahmens }\end{array}$ \\
\hline $\begin{array}{l}\text { Stabilität des Europäischen } \\
\text { Finanzmarkts }\end{array}$ & $\begin{array}{l}\text { Weiterentwicklung der Europäischen Finanzmarktaufsicht, } \\
\text { Schaffung gemeinsamer Aufsichtsinstrumente zur } \\
\text { Sanierung von Finanzinstituten }\end{array}$ \\
\hline
\end{tabular}




\begin{tabular}{|c|c|}
\hline Ziel & Vorgesehene Maßnahmen zur Umsetzung \\
\hline \multicolumn{2}{|c|}{ Wettbewerbsfähigkeit - Haushalts- und Wirtschaftspolitik } \\
\hline Makroökonomische Stabilität & Verbesserung wirtschaftspolitischer Abstimmungsprozesse \\
\hline Haushaltskonsolidierung & Abbau des Schuldenstandes, Investitionen in Lissabonziele \\
\hline $\begin{array}{l}\text { Verringerung der Belastung des } \\
\text { Faktors Arbeit }\end{array}$ & $\begin{array}{l}\text { Prüfung und ggf. Senkung der Steuer- und Abgabenlast, } \\
\text { vorrangige Entlastung Geringqualifizierter }\end{array}$ \\
\hline \multicolumn{2}{|c|}{ Sozialer Zusammenhalt - Bildung und Ausbildung } \\
\hline $\begin{array}{l}\text { Koordination der bildungs- } \\
\text { politischen Planungen }\end{array}$ & $\begin{array}{l}\text { Erstellung eines „Europäische Pakts für die Jugend“, } \\
\text { Halbierung der Zahl der 18- bis 24-jährigen mit } \\
\text { Sekundarstufe I als höchstem Bildungsabschluss }\end{array}$ \\
\hline Schaffung lokaler Lernzentren & $\begin{array}{l}\text { Ausbau der Schulen, Förderung von Lernpartnerschaften } \\
\text { zw. Schulen, Unternehmen und Forschungseinrichtungen }\end{array}$ \\
\hline $\begin{array}{l}\text { Förderung der IKT- } \\
\text { Grundfähigkeiten }\end{array}$ & $\begin{array}{l}\text { Finanzielle Investitionen, Ausbau von Schulungen und } \\
\text { anderen Weiterbildungsmaßnahmen }\end{array}$ \\
\hline \multicolumn{2}{|c|}{ Sozialer Zusammenhalt - Aktive Beschäftigungspolitik } \\
\hline $\begin{array}{l}\text { Steigerung der Gesamterwerbs- } \\
\text { tätigenquote auf } 70 \% \text {, bei Frauen auf } \\
60 \% \text {, bei } 55-64 \text { jährigen auf } 50 \% \text {; } \\
\text { Förderung von Weiterbildungs- } \\
\text { maßnahmen }\end{array}$ & $\begin{array}{l}\text { Schaffung einer europaweiten Datenbank für Stellen und } \\
\text { Lernangebote, benchmarks für lebenslanges Lernen }\end{array}$ \\
\hline $\begin{array}{l}\text { Förderung von Chancengleichheit, } \\
\text { Vereinbarkeit von Familie und Beruf }\end{array}$ & benchmarks für Kinderbetreuungsmaßnahmen \\
\hline \multicolumn{2}{|c|}{ Sozialer Zusammenhalt - Modernisierung der sozialen Sicherungssysteme } \\
\hline $\begin{array}{l}\text { Angemessene Entlohnung, } \\
\text { Vorbereitung auf die Auswirkungen } \\
\text { des demographischen Wandels }\end{array}$ & $\begin{array}{l}\text { Einrichtung einer high-level group zum Thema „Sozial- } \\
\text { schutz“, Erstellung einer Studie zur Tragfähigkeit der } \\
\text { Altersversorgungssysteme, Nutzung der OMK }\end{array}$ \\
\hline \multicolumn{2}{|c|}{ Sozialer Zusammenhalt - Soziale Integration } \\
\hline $\begin{array}{l}\text { Wohlstand und soziale Teilhabe } \\
\text { durch Integration in die } \\
\text { Informationsgesellschaft }\end{array}$ & $\begin{array}{l}\text { Berücksichtigung des Ziels der sozialen Integration in allen } \\
\text { relevanten Politikbereichen, Neuausrichtung der Struktur- } \\
\text { fonds, Nutzung der OMK }\end{array}$ \\
\hline \multicolumn{2}{|c|}{ Sozialer Zusammenhalt - Umweltschutz } \\
\hline $\begin{array}{l}\text { Anteil Erneuerbarer Energien an der } \\
\text { Stromerzeugung i.H.v. } 21 \%\end{array}$ & \multirow{3}{*}{$\begin{array}{l}\text { Änderung des Rechtsrahmens, finanzielle Förderung, } \\
\text { Bereitstellung von Mitteln der EIB und des EIF, Nutzung } \\
\text { der OMK }\end{array}$} \\
\hline $\begin{array}{l}\text { Anteil der Kraft-Wärme-Kopplung } \\
\text { an der Stromgewinnung i.H.v. } 18 \%\end{array}$ & \\
\hline $\begin{array}{l}\text { Minderung des Treibhausgasaus- } \\
\text { stoßes gegenüber } 1990 \text { um } 8 \%\end{array}$ & \\
\hline
\end{tabular}

Quelle: Eigene Darstellung nach Europäischer Rat: Schlussfolgerungen des Vorsitzes, Brüssel, 22. und 23.03.2005.

\section{Ergebnisse}

Zwei Ereignisse sind bei der Bewertung der Lissabon-Strategie zu berücksichtigen: die EU-Erweiterungsrunden 2004 und 2007 sowie die Finanz- und Wirtschaftskrise, die bereits 2008, vor allem aber 2009 auf die Mitgliedstaaten einwirkte. Beide Entwicklungen verzerren die statistischen Daten in Teilen 
beträchtlich. Zudem ist zu beachten, dass eine abschließende Bewertung - trotz der Diskussion um die neue Strategie „Europa 2020“ - erst anhand der Daten für das Referenzjahr 2010 möglich sein wird; deren Veröffentlichung ist für 2011 vorgesehen. Die nachfolgend wiedergegebenen Statistiken beziehen sich auf die Jahre 2008 sowie 2009 und decken - unter der Vorgabe einer Gesamtbewertung der Lissabon-Strategie - die ursprünglichen EU-15 sowie in Teilen (und zur vergleichenden Betrachtung) die EU-27 ab.

In der Summe ergibt sich - ohne der detaillierten Betrachtung vorgreifen zu wollen - ein nahezu durchgehend ambivalentes Bild: Während zahlreiche der vorgesehenen benchmarks verfehlt wurden, sind in einer Reihe von Aufgabenfeldern deutliche Verbesserungen der Ausgangssituation zu verzeichnen. Die Entwicklung verlief je nach Zielvorgabe und Mitgliedstaat (erwartbar) unterschiedlich; die Distanz zwischen den ,erfolgreichsten“ und den weniger aktiven Mitgliedstaaten vergrößerte sich in Teilen beträchtlich. ${ }^{11}$

Im Einzelnen lassen sich mit Blick auf die in Tab. 1 vorgestellte Gliederung folgende Ergebnisse erkennen:

\section{Wettbewerbsfähigkeit}

\section{a) Informationsgesellschaft}

In diesem Bereich konnte eine Reihe von Fortschritten erzielt werden, wobei v.a. im Rahmen der neu geschaffenen eEurope-Aktionspläne bestehende Maßnahmen der Gemeinschaft wie der Mitgliedstaaten gebündelt, aufeinander bezogen und durch neue Vorhaben ergänzt wurden. Die Umsetzung erfolgte dadurch zielgerichteter; die Förderung der privaten und öffentlichen IKT-Nutzung verlief in vielen Fällen erfolgreich. So verfügten bereits 2006 etwa 97\% aller Schulen in der EU über einen Internetanschluss, davon zwei Drittel über eine schnelle Breitbandverbindung. ${ }^{12}$ Auch der Anteil der online angebotenen Dienstleistungen der öffentlichen Verwaltung (eGovernment-Dienste) wurde deutlich ausgeweitet: von $36 \%$ (2002) auf $81 \%(2009)^{13}$ für die EU-15, in der EU-27 auf immerhin 74\%. Die Nutzung dieser Angebote durch Unternehmen stieg parallel

11 Tilford, S./Whyte, Ph.: The Lisbon Scorecard X - The Road to 2020, London: Centre for European Reform, 2010, 3.

12 Europäische Kommission: Europaweite Umfrage - mehr als zwei Drittel der EU-Schulen verfügen über schnellen Internetzugang. IP/06/1285, Brüssel, 29.09.2006.

13 Die Daten beziehen sich auf die EU-15. Eurostat: Online-Verfügbarkeit des e-Government in Prozent der Online-Verfügbarkeit von 20 grundlegenden öffentlichen Diensten, 15.10.2010. 
dazu von 51\% (2004) auf 72\% (2009, EU-15) an. ${ }^{14}$ Nur unwesentliche Verbesserungen konnten hingegen in den Bereichen elektronischer Geschäftsverkehr Nutzungssteigerung von 9\% (2004) auf 13\% (2009) ${ }^{15}$ - und eCommerce - von $0,9 \%$ (2002) auf 4,4\% (2007) ${ }^{16}$ - verzeichnet werden. Die Versorgung der Gesamtbevölkerung mit schnellen Internetverbindungen stieg von 2,3\% (2002) auf 26,4\% (2009, EU-15), für die EU-27 auf bereits 23,9\% (2009). ${ }^{17}$

Während die Liberalisierung der nationalen Telekommunikationsmärkte bereits weit vorangeschritten ist, kann von einem unionsweit integrierten Markt bislang nicht die Rede sein. Als ursächlich erwiesen sich elementare Unterschiede zwischen den mitgliedstaatlichen Regelungsformen und -inhalten sowie die zwar rückläufigen, jedoch weiterhin beträchtlichen Marktanteile der überkommenen Monopoldienstleister. ${ }^{18}$ Im Mobilfunkmarkt fiel der Anteil der Marktführer $a b$ ovo geringer aus und sank von $48 \%$ (2001) auf $37 \%$ (2009). ${ }^{19}$ Anhand der Entwicklung der Verbindungspreise können die Liberalisierungserfolge im Inland und die weiterhin bestehenden Rigiditäten im EU-Raum vergleichend untersucht werden: Danach wurden die Kosten für Inlandstelefonate deutlich reduziert, während für EU-weite Gespräche weiterhin hohe Preise anfallen - mit beträchtlichen Unterschieden zwischen den Mitgliedstaaten. ${ }^{20}$ Mit Blick auf die sog. roaming-Kosten griff die Kommission allerdings zwischenzeitlich regulierend ein. Das Frequenzangebot für Mobilkommunikationssysteme schließlich verbesserte sich im gleichen Zeitraum; so wurden die Mobilfunknetze im Jahr 2009 unionsweit harmonisiert und für drahtlose Breitbanddienste (UMTS) geöffnet, ${ }^{21}$ während die Mitgliedstaaten in Teilen bereits dazu übergegangen sind, neue Übertragungstechnologien auf nationaler Ebene zu implementieren.

14 ders.: Nutzung des e-Government durch Unternehmen, 15.10.2010.

15 ders.: Anteil des elektronischen Geschäftsverkehrs am Gesamtumsatz der Unternehmen, 15.10.2010.

16 Die Daten beziehen sich auf die EU-15; ders.: E-Commerce via Internet als Anteil des elektronischen Geschäftsverkehrs am Gesamtumsatz der Unternehmen, 15.10.2010.

17 Die Daten beziehen sich auf die EU-15; ders.: Versorgungsgrad mit Breitbandanschlüssen als Anzahl der Breitbandanschlüsse je 100 Einwohner, 15.10.2010.

18 Tilford, S./Whyte, Ph., a.a.O., 29; der Marktanteil der ehemaligen Staatsbetriebe sank im EUDurchschnitt von 82\% (2002) auf 69\% (2005). Diese Daten beziehen sich auf die EU-15; Eurostat: Marktanteil des traditionell etablierten Anbieters im Festnetz nach Art des Gesprächs, 15.10.2010.

19 Die Daten beziehen sich auf die EU-15; ders.: Markanteil des führenden Anbieters im Mobilnetz in Prozent des Gesamtmarktes, 15.10.2010.

20 ders.: Preise für Telekommunikation nach Art des Gesprächs, 15.10.2010.

21 Europäisches Parlament/Rat: Richtlinie 2009/114/EG; Europäische Kommission: Entscheidung 2009/766/EG. 


\section{b) Europäischer Forschungsraum}

Für den „Europäische Forschungsraum“ (EFR) galt als übergeordnetes Ziel, eine „fünfte Grundfreiheit“ des EU-Binnenmarktes zu schaffen: den freien Verkehr von Wissen. Der Weg dorthin wurde zwar durch zahlreiche Initiativen vorgezeichnet, bislang aber nur in Ansätzen beschritten. Als Kern des ERF galten die sog. Europäischen Technologieplattformen (ETF), auf deren Basis die Forschungsbemühungen in unterschiedlichen Handlungsfeldern einer verbesserten Koordination und effizienteren Gestaltung zugeführt werden sollten; ${ }^{22}$ zur Finanzierung grenzüberschreitender Forschungsprojekte beschloss man 2006 mit dem 7. EU-Forschungsrahmenprogramm die Bereitstellung eines bislang einmaligen Ressourcenvolumens i.H.v. 53,3 Mrd. Euro bis 2013. ${ }^{23}$ Zudem wurden die Mittel der EIB zur Förderung von Innovationen und KMU aufgestockt, ${ }^{24}$ die Kohäsionspolitik auf Wachstum, Beschäftigung und die Förderung von KMU ausgerichtet sowie Innovationen in den Bereichen Energieeffizienz und Erneuerbare Energien gesondert gefördert. ${ }^{25}$ Das im Jahr 2008 gegründete Europäische Institut für Innovation und Technologie $(E I T)^{26}$ soll diese Bemühungen in Zukunft bündeln und an die nachfragende Wissenschaft vermitteln, um lokale cluster, internationale Netzwerke sowie den Austausch zwischen Universitäten, Forschungseinrichtungen und Unternehmen zu begünstigen. ${ }^{27}$ Der inzwischen veröffentlichte „Europäische Innovationsanzeiger“ berichtet regelmäßig über die Fortentwicklung des EFR.

Die statistisch konkretisierten Zielsetzungen, allen voran die Anhebung der FuEAusgaben auf 3\% des Bruttoinlandsprodukts (GERD), wurden hingegen weitgehend verfehlt: Das GERD stieg im EU-27-Durchschnitt von 1,74\% (2000) auf lediglich 1,81\% (2008) des BIP. ${ }^{28}$ Die 3-Prozent-Marke konnte nur in Schweden und Finnland erreicht werden, jedoch ist hier kaum eine Verbindung zur Lissa-

23 Europäisches Parlament: Beschluss Nr. 1982/2006/EG; Rat: Richtlinie Nr. 969/2006/EG; Europäisches Parlament/Rat: Beschluss Nr. 1639/2006/EG.

24 Europäische Kommission: $\operatorname{KOM}(2008) 800,7$.

25 dies.: KOM (2005) 330, 5.

26 Europäisches Parlament/Rat: Verordnung (EG) Nr. 294/2008.

27 Vgl. Europäische Kommission: $\operatorname{KOM}(2008)$ 881, 7.

28 OECD: Bruttoinlandsausgaben für FuE (GERD) in Prozent des BIP, 10.12.2010; für die EU-15 liegen die entsprechenden Werte bei $1,85 \%$ bzw. 1,95\%. Eurostat verzeichnet im gleichen Zeitraum Werte von 1,86\% bzw. 1,92\% für die EU-27 und schätzt, dass im Jahr 2009 die 2-Prozent-Marke durchbrochen wurde $(2,01 \%)$. Aus Gründen der außereuropäischen Vergleichbarkeit werden die Daten der OECD zitiert. 
bon-Strategie zu unterstellen: Schweden verzeichnete schon 1993 einen Wert von 3,12\% und auch Finnland hatte den Zielwert bereits vor Beginn des Planungszeitraums der Strategie erreicht (3,17\% im Jahr 1999). Eine Reihe mittelund osteuropäischer Mitgliedstaaten meldeten indes Werte i.H.v. weniger als $1 \%$ des BIP (vgl. Abb. 1).

Abbildung 1: Bruttoinlandsausgaben für FuE (GERD) in Prozent des BIP (2008)

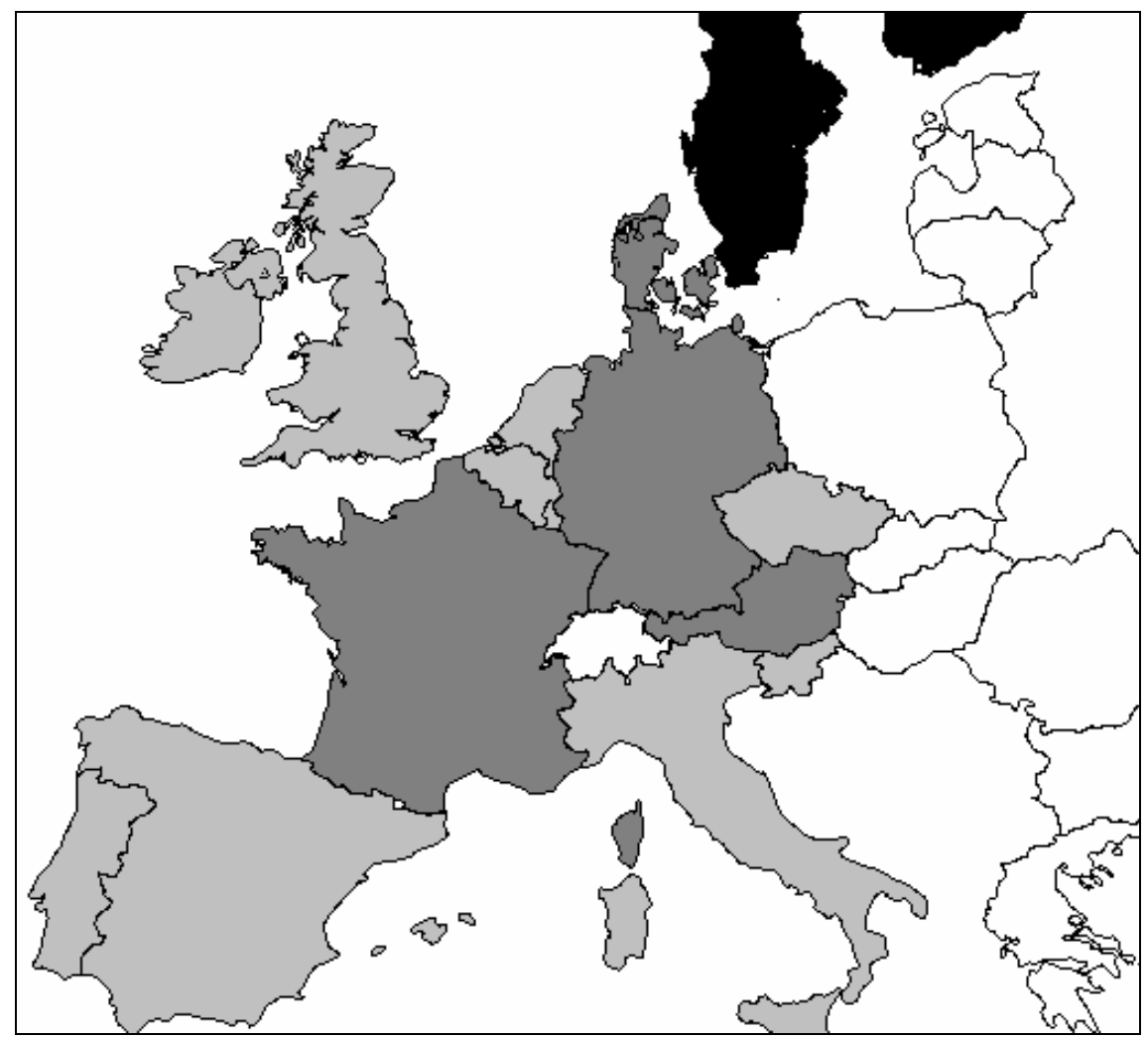

Legende: EU-27 (aus Darstellungsgründen exkl. Zypern und Malta); schwarz - 3,0 bis 3,48\%; dunkelgrau - 2,0 bis 3,0\%; hellgrau - 1,0 bis 2,0\%; weiß - 0,46 bis 1,0\%. Quelle: OECD: Bruttoinlandsausgaben für FuE (GERD) in Prozent des BIP, 10.12.2010.

Zwar wird zurecht darauf verwiesen, dass sich das GERD nur bedingt zur Messung der Intensität von Forschung und Entwicklung eignet, da Innovationen im Dienstleistungssektor (der die Wirtschaftsstruktur der EU bereits seit Jahrzehnten 
dominiert) nicht vollständig in die Berechnungen einfließen, ${ }^{29}$ doch relativiert dies kaum die erkennbaren Fehlentwicklungen: Der Abstand etwa zu den USA (2008: $2,66 \%)$ und Japan $(3,44 \%)$ besteht weiterhin, ${ }^{30}$ der finanzielle und bürokratische Aufwand für einen EU-weiten Patentschutz ist nach wie vor beträchtlich und das avisierte Gemeinschaftspatent harrt noch immer der Umsetzung. ${ }^{31}$

Höchst unterschiedlich fielen die steuerpolitischen Maßnahmen der Mitgliedstaaten zur Umsetzung der Innovationspolitik aus, ein unionsweiter Ansatz findet sich auch hier bislang nicht. Die Kommission sah entsprechende Anreizpolitiken zwar als ,zielführendes Instrument“ und veröffentlichte regelmäßig Anregungen zur Gestaltung und Umsetzung mitgliedstaatlicher Maßnahmen, doch fanden diese kaum nennenswerte Beachtung in den europäischen Hauptstädten. ${ }^{32}$

Auch die Bemühungen, mehr junge Menschen für ein Studium der Natur- und Technikwissenschaften zu gewinnen, waren in nur begrenztem Maße erfolgreich: Im europäischen Durchschnitt stieg der Anteil der natur- und technikwissenschaftlichen Fächer an den Abschlüssen im tertiären Bildungsbereich von 10,1\% (2002) auf $13,9 \%(2008))^{33}$

Altbewährtes - etwa die SOKRATES-Programme samt der inzwischen fest etablierten ERASMUS-Förderung studentischer Auslandsaufenthalte - wurde vergleichsweise häufig in Anspruch genommen; die Mobilität von Studenten und Wissenschaftlern wuchs in einigen Mitgliedstaaten spürbar, ohne jedoch einen einheitlichen Trend zu konstituieren (vgl. Abb. 2). ${ }^{34}$ Zahlreiche Initiativen der Kommission zur Förderung der beruflichen Mobilität, wie etwa ein entsprechender Europäischer Aktionsplan, ${ }^{35}$ das EU-weite Arbeitsplatzvermittlungssystem EURES sowie zielgruppenspezifische Portale für Forscher und einzelne Berufsgruppen erzielten hingegen kaum Breitenwirkung:

So stammen nur etwa $2 \%$ der Arbeitskräfte in den EU-25-Ländern aus einem anderen Mitgliedstaat; ${ }^{36}$ eine repräsentative Eurobarometer-Umfrage ergab zu-

29 Tilford, S./Whyte, Ph., a.a.O., 22.

30 OECD: Bruttoinlandsausgaben für FuE (GERD) in Prozent des BIP, 10.12.2010.

31 Tilford, S./Whyte, Ph., a.a.O., 22.

32 Europäische Kommission: $\operatorname{KOM}(2008) 881,9$.

33 Eurostat: Tertiärabschlüsse in naturwissenschaftlichen und technologischen Fachrichtungen, 15.10.2010.

34 ders.: Jährliche Daten über die Arbeitsplatzmobilität von erwerbstätigen HRST, 25-64 Jahre, 15.10.2010.

35 Europäische Kommission: $\operatorname{KOM(2007)~} 773$.

36 Europäischer Wirtschafts- und Sozialausschuss: 2009/C 228/03, C228/17. 
dem, dass zwar 17\% der Bevölkerung ggf. im EU-Ausland arbeiten würden, der EURES-Mechanismus aber nur jedem fünfzigsten Befragten bekannt war. ${ }^{37} \mathrm{Im}$ Zeitraum zwischen 2000 und 2006 unterstützte der Europäische Sozialfonds jährlich etwa 10 Mio. Menschen in migrationsrelevanten Fragen, doch waren bislang nur jene Förderlinien, die sich an die Studentenschaft richteten, ansatzweise erfolgreich. $^{38}$

Abbildung 2: Mobilität von Studenten in Europa (2007)

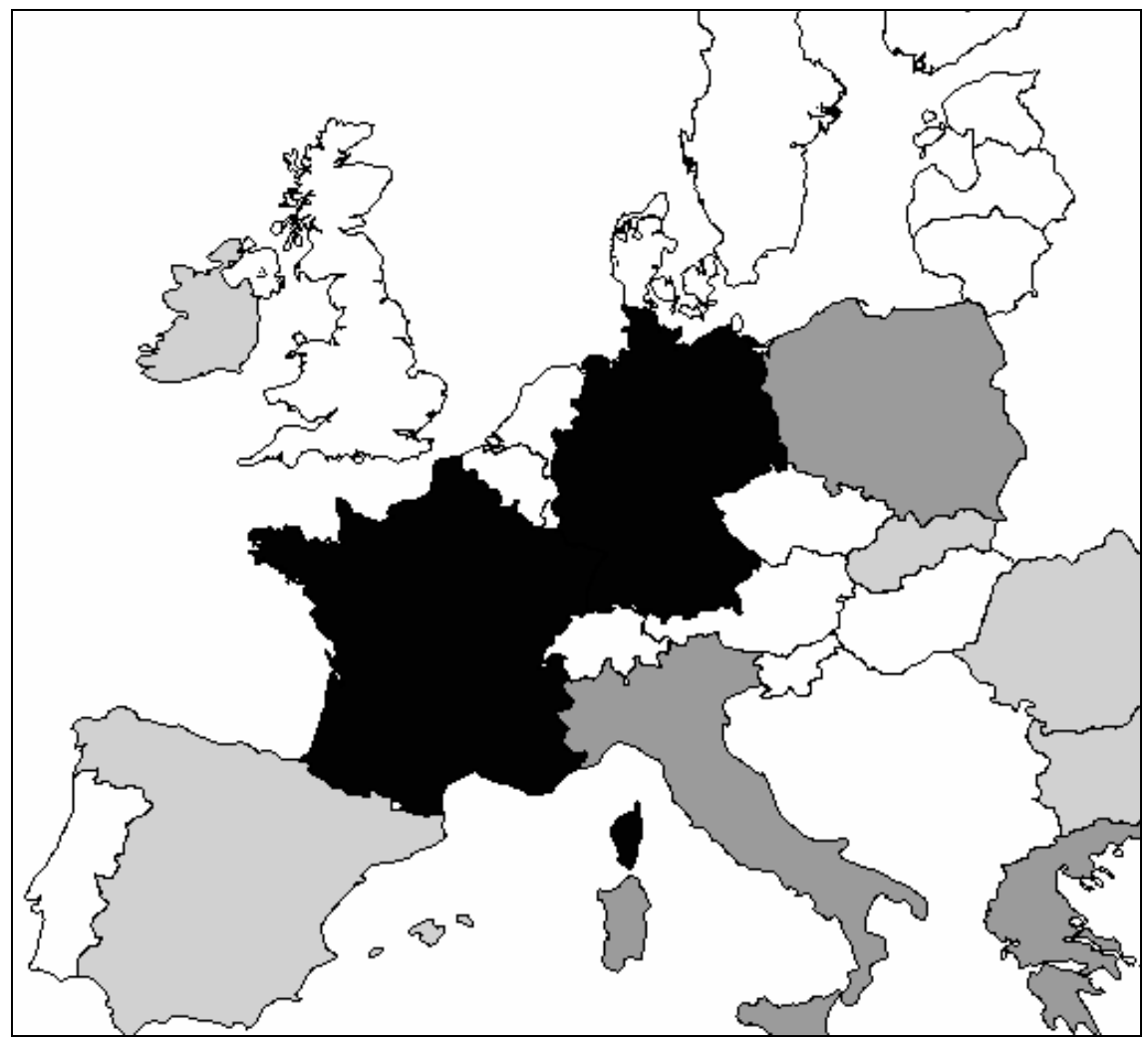

Legende: EU-27 (aus Darstellungsgründen exk1. Zypern und Malta); Prozentsatz der Studierenden, die in anderen EU-Mitgliedstaaten studieren; schwarz - 40,0 bis 65,4\%; dunkelgrau - 30 bis $40 \%$; hellgrau - 15 bis 30\%; weiß - 1,0 bis 15\%. Quelle: Eurostat: Mobilität von Studenten in Europa Studierende (ISCED 5-6), die in anderen EU-Mitgliedstaaten studieren, 15.10.2010.

37 Europäische Kommission: Special Eurobarometer 337 "Geographical and labour mobility" March 2010.

38 dies.: The European Job Mobility Action Plan 2007-2010 - Implementation Report, 7. 
Als weitgehend angenommen gilt der „Europass“; er ermöglicht die EU-weite Vergleichbarkeit von Qualifikationen wie Bildungsabschlüssen, Sprachkenntnissen und beruflichen Erfahrungen. Die wechselseitige Anerkennung von Abschlüssen blieb allerdings unzureichend: So wurden im EU-Durchschnitt $30 \%$ aller der Kommission gemeldeten Anträge auf Anerkennung von Berufsqualifikationen ganz oder teilweise abgelehnt, ${ }^{39}$ viele Interessenten scheiterten an den bürokratischen Hürden, die sich aus dem vorhandenen Geflecht von gemeinschaftlichen und mitgliedstaatlichen Bestimmungen ergeben. Aufgrund der bislang eher zögerlichen Umsetzung der Richtlinie über die Anerkennung von Berufsqualifikationen verbleiben beträchtliche Unterschiede zwischen den einzelnen Mitgliedstaaten. ${ }^{40}$ Schließlich stellt die Öffnung der Arbeitsmärkte Deutschlands, Großbritanniens und Österreichs für Arbeitskräfte aus den neuen Mitgliedstaaten eine dringliche, bislang ungelöste Aufgabe dar.

\section{c) Standortbedingungen für privatwirtschaftliche Unternehmen}

Die Ausgangsbedingungen für unternehmerische Tätigkeit verbesserten sich insgesamt nur marginal, obwohl einige Mitgliedstaaten - v.a. die Gruppe der „Nachzügler“ - eine Reihe von Fortschritten erzielen konnten. ${ }^{41}$ Entsprechende Ziele und Leitlinien wurden im Jahr 2000 durch eine „Europäische Charta für Kleinunternehmen" formuliert, ${ }^{42}$ in deren Gefolge die Kommission v.a. den KMU-Bereich mit zahlreichen Initiativen zu fördern begann; auch die Mittelvergabe von EIB und EIF wurde zugunsten von KMU neu ausgerichtet. Im Jahr 2008 legte die Kommission mit dem Small Business Act zudem ein ehrgeiziges Maßnahmenprogramm vor: Im Zentrum standen Änderungen der bestehenden Beihilferegeln zugunsten kleiner und mittelständischer Unternehmen, verbunden mit der Anwendung ermäßigter Mehrwertsteuersätze auf deren Produkte. Diese Maßnahmen änderten jedoch wenig an den weiterhin hohen Kosten, die sich in den meisten Mitgliedstaaten mit einer Unternehmensgründung verbinden, und dem noch immer unzureichenden Angebot an entsprechendem Risikokapital (das weit unter dem Niveau der in diesem Bereich führenden USA liegt). ${ }^{43}$

40 Europäisches Parlament/Rat: Richtlinie 2005/36/EG.

41 Tilford, S./Whyte, Ph., a.a.O., 46.

42 Europäischer Rat: Schlussfolgerungen des Vorsitzes. ANLAGE III - Europäische Charta für Kleinunternehmen. 19. und 20.06.2000.

43 Tilford, S./Whyte, Ph., a.a.O., 45. 
Hohe Bürokratiekosten und unterschiedliche nationale Rechtsvorschriften stellen die größten Hindernisse für Unternehmensgründungen dar. ${ }^{44}$ Bislang haben nur einzelne Mitgliedstaaten Maßnahmen zur Rückführung des im internationalen Vergleich äußerst hohen Verwaltungsaufwands umgesetzt; ein gemeinsames Vorgehen ist nicht erkennbar. Auch mangelt es - trotz langjähriger Verhandlungen - weiterhin an einer europaweit einheitlichen Rechtsform für KMU; die Einführung einer entsprechenden Regelung für Aktiengesellschaften (Societas Europaea, SE) wird hingegen als Erfolg eingeschätzt.

Die Tätigkeit der Kommission in diesem Bereich weist somit eine eher ,durchwachsene“ Erfolgsbilanz auf: Zwar konnte die Umsetzung von binnenmarktrelevantem Gemeinschaftsrecht in Teilen deutlich verbessert werden - im EUDurchschnitt auf 99,1\% ${ }^{45}$-, doch sank die Zahl der Vertragsverletzungsverfahren im gleichen Zeitraum kaum, obwohl die im Jahr 2002 neu eingerichtete Vermittlungsstelle SOLVIT inzwischen zahlreiche Beschwerden von Unternehmen und Privatpersonen (etwa $80 \%$ der bei SOLVIT anhängigen Verfahren ${ }^{46}$ ) ohne Beteiligung der Kommission bearbeitet. ${ }^{47}$ Auch in diesem Bereich finden sich zudem deutliche Unterschiede zwischen den Mitgliedstaaten; die personelle, finanzielle und materielle Ausstattung der nationalen SOLVIT-Stellen obliegt den nationalstaatlichen Regierungen.

Maßnahmen zur Reduzierung der bereits angesprochenen Bürokratiekosten waren kaum erfolgreich, da sich die Überprüfung des gesamten acquis als äußerst arbeitsintensiv erwies und die Untersuchungen der dafür eingesetzten High Level Group of Independent Stakeholders on Administrative Burdens bislang kaum belastbare Ergebnisse zeitigten. ${ }^{48}$ Das Gemeinschaftsprogramm „Better Regulation" richtete sich neben der Vereinfachung und Systematisierung von Rechtsvorschriften auch auf eine Verbesserung der Folgenabschätzung, eine Verringerung des Verwaltungsaufwands und eine intensivere Einbindung der betroffenen stakeholder. Der Kommission zufolge konnte der Umfang des acquis auf diese Weise zwar um etwa $10 \%$ reduziert werden, als langfristig bedeutsamer dürften

44 dies., 46.

45 Im Jahr 2010; vgl. Europäische Kommission: Binnenmarktanzeiger September 2010.

46 Tilford, S./Whyte, Ph., a.a.O., 54.

47 Europäische Kommission: 27. Jahresbericht über die Kontrolle der Anwendung des EU-Rechts. 2009.

48 High Level Group of Independent Stakeholders on Administrative Burdens: Intermediate Report of the High Level Group of Independent Stakeholders on Administrative Burdens - Achievements and challenges. 17.09.2009; vgl. kritisch hierzu Hesse, J.J.: Das Regierungssystem der Bundesrepublik Deutschland, 10. Auflage, 2011 (i.E.), Kapitel V. 
sich jedoch kostenrelevante Änderungen im Bereich der unternehmerischen Meldepflichten erweisen. ${ }^{49}$

Zusammenfassend lohnt ein Blick in den Jahresbericht Doing Business der Weltbank, der die Attraktivität einzelner Staaten für Unternehmensgründungen und andere Geschäftstätigkeiten zu analysieren sucht. ${ }^{50}$ Unter den top ten finden sich mit Ausnahme Großbritanniens, Dänemarks und Irlands keine EUMitgliedstaaten. Die meisten von ihnen rangieren im Mittelfeld, während einige als besonders unternehmensunfreundlich gelten (vgl. Tab. 2). Dass die Liberalisierung der Märkte v.a. in Irland erkennbar nur in Teilen vom Gedanken der Nachhaltigkeit geprägt war, sei mit Blick auf die aktuelle Entwicklung hinzugefügt.

Tabelle 2: „Ease of Doing Business“ (top ten plus EU-Mitgliedstaaten, 2009)

\begin{tabular}{l|c|c|c|c|c}
\hline Economy & Rank & $\begin{array}{c}\text { Starting a } \\
\text { Business }\end{array}$ & $\begin{array}{c}\text { Employing } \\
\text { Workers }\end{array}$ & $\begin{array}{c}\text { Getting } \\
\text { Credit }\end{array}$ & Paying Taxes \\
\hline Singapore & $\mathbf{1}$ & 4 & 1 & 4 & 5 \\
\hline New Zealand & $\mathbf{2}$ & 1 & 15 & 4 & 9 \\
\hline Hong Kong SAR & $\mathbf{3}$ & 18 & 6 & 4 & 3 \\
\hline United States & $\mathbf{4}$ & 8 & 1 & 4 & 61 \\
\hline United Kingdom & $\mathbf{5}$ & 16 & 35 & 2 & 16 \\
\hline Denmark & $\mathbf{6}$ & 28 & 9 & 15 & 13 \\
\hline Ireland & $\mathbf{7}$ & 9 & 27 & 15 & 6 \\
\hline Canada & $\mathbf{8}$ & 2 & 17 & 30 & 28 \\
\hline Australia & $\mathbf{9}$ & 3 & 1 & 4 & 47 \\
\hline Norway & $\mathbf{1 0}$ & 35 & 114 & 43 & 17 \\
\hline Finland & $\mathbf{1 6}$ & 30 & 132 & 30 & 71 \\
\hline Sweden & $\mathbf{1 8}$ & 43 & 117 & 71 & 42 \\
\hline Belgium & $\mathbf{2 2}$ & 31 & 48 & 43 & 73 \\
\hline Estonia & $\mathbf{2 4}$ & 37 & 161 & 43 & 38 \\
\hline Germany & $\mathbf{2 5}$ & 84 & 158 & 15 & 71 \\
\hline Lithuania & $\mathbf{2 6}$ & 99 & 119 & 43 & 51 \\
\hline Latvia & $\mathbf{2 7}$ & 51 & 128 & 4 & 45 \\
\hline Austria & $\mathbf{2 8}$ & 122 & 60 & 15 & 102 \\
\hline Netherlands & $\mathbf{3 0}$ & 70 & 123 & 43 & 33 \\
\hline France & $\mathbf{3 1}$ & 22 & 155 & 43 & 59 \\
\hline Cyprus & $\mathbf{4 0}$ & 25 & 93 & 71 & 37 \\
\hline Slovak Republic & $\mathbf{4 2}$ & 66 & 81 & 15 & 119 \\
\hline Bulgaria & $\mathbf{4 4}$ & 50 & 53 & 4 & 95 \\
\hline Hungary & $\mathbf{4 7}$ & 39 & 77 & 30 & 122 \\
\hline Portugal & $\mathbf{4 8}$ & 60 & 171 & 87 & 80 \\
\hline Slovenia & $\mathbf{5 3}$ & 26 & 162 & 87 & 84 \\
\hline & & & & & \\
\hline
\end{tabular}

49 Europäische Kommission: $\operatorname{KOM}(2009)$ 15, 3.

50 World Bank: Doing Business, Washington D.C., 2009. 


\begin{tabular}{l|c|c|c|c|c}
\hline Economy & Rank & $\begin{array}{c}\text { Starting a } \\
\text { Business }\end{array}$ & $\begin{array}{c}\text { Employing } \\
\text { Workers }\end{array}$ & $\begin{array}{c}\text { Getting } \\
\text { Credit }\end{array}$ & Paying Taxes \\
\hline Romania & $\mathbf{5 5}$ & 42 & 113 & 15 & 149 \\
\hline Spain & $\mathbf{6 2}$ & 146 & 157 & 43 & 78 \\
\hline Luxembourg & $\mathbf{6 4}$ & 72 & 170 & 113 & 15 \\
\hline Poland & $\mathbf{7 2}$ & 117 & 76 & 15 & 151 \\
\hline Czech Republic & $\mathbf{7 4}$ & 113 & 25 & 43 & 121 \\
\hline Italy & $\mathbf{7 8}$ & 75 & 99 & 87 & 136 \\
\hline Greece & $\mathbf{1 0 9}$ & 140 & 147 & 87 & 76 \\
\hline
\end{tabular}

Quelle: World Bank: Doing Business, 2009.

\section{d) Binnenmarkt für Dienstleistungen; Beihilfepolitik}

Die Entwicklung des Binnenmarktes im Dienstleistungssektor ist von besonderer Bedeutung, da dieser Wirtschaftszweig inzwischen mehr als 70\% des BIP der Union erwirtschaftet. ${ }^{51}$ Durch die im Jahr 2010 in Kraft getretene Dienstleistungsrichtlinie konnten Fortschritte bei der Liberalisierung erzielt werden. ${ }^{52}$ Neben der Anpassung der nationalen Rechtsrahmen sind die „Einheitlichen Ansprechpartner" ein Kernbestandteil der Umsetzung. Sie dienen als zentrale nationale Beratungsstellen ausländischer Unternehmen und informieren über alle geschäftsrelevanten Angelegenheiten.

Die einzelnen Dienstleistungsbereiche entwickelten sich indessen durchaus unterschiedlich. So blieb v.a. der Energiesektor weitgehend von effektiven Liberalisierungsmaßnahmen verschont: Zwar wurde im Jahr 2003 der Aufbau nationaler Regulierungsbehörden beschlossen, die den Wettbewerb und hier v.a. den Zugang zu den Netzinfrastrukturen überwachen sollten, ${ }^{53}$ doch konnten mit Blick auf den gesamteuropäischen Energiemarkt bislang nur unter Rückgriff auf die verfügbaren kartellpolitischen Instrumente nennenswerte Ergebnisse erzielt werden. Einige Anbieter mussten sich von ihren Strom- bzw. Gasnetzen trennen, um hohe Strafzahlungen zu vermeiden; 2009 wurde die formale Trennung von Netz und Anbietern beschlossen ${ }^{54}$ - allerdings mit einem ,Schlupfloch“ insofern, als es den Versorgern gestattet wurde, die Netze lediglich in eine Tochtergesell-

51 Eurostat: Bruttowertschöpfung - Handel, Transport und Nachrichtenübermittlung, 15.10.2010; Eurostat: Bruttowertschöpfung - Unternehmensbezogene und Finanzdienstleistungen, 15.10.2010; Eurostat: Bruttowertschöpfung - Sonstige Dienstleistungen, 15.10.2010.

52 Europäisches Parlament/Rat: Richtlinie 2006/123/EG.

53 dies.: Richtlinie 2003/54/EG; dies.: Richtlinie 2003/55/EG.

54 dies.: Verordnung Nr. 714/2009; dies.: Verordnung Nr. 715/2009; dies.: Richtlinie 2009/72/EG; dies.: Richtlinie 2009/73/EG. 
schaft zu überführen. Die weiterhin bestehenden Oligopole einiger weniger Anbieter blieben im Grundsatz unangetastet. ${ }^{55}$

Der Transportsektor erfuhr hingegen eine in Teilen deutliche Liberalisierung: Während der Markt in der Luftverkehrsbranche bereits als weitgehend integriert galt, konnten nun auch im Bereich des Schienenverkehrs deutliche Fortschritte erzielt werden. Die Umsetzung der umfangreichen Maßnahmenpakete zur Deregulierung des Güter- und Personenverkehrs erfolgte seitens der Mitgliedstaaten allerdings nur stockend. Während nationalstaatliche Rechtsvorschriften in einigen Ländern den Markteintritt ausländischer Unternehmen grundsätzlich behindern, ${ }^{56}$ scheitert der konsequente Ausbau transnationaler Schienenverbindungen v.a. an den hohen Kosten. ${ }^{57}$ Ein grundsätzlicher Konflikt im Transportsektor besteht zudem zwischen den beiden „Lissabonzielen“ der ökonomischen Effizienz und der ökologischen Nachhaltigkeit. So ist der Transportsektor der europaweit einzige Industriebereich, der in den vergangenen Jahren einen deutlichen Anstieg der verursachten Treibhausgasemissionen verzeichnete. ${ }^{58}$

Die Postdienstleistungen wurden zwar formal liberalisiert, insbesondere im Brieftransport erschweren jedoch nationalstaatliche Regulierungen (die bis spätestens 2012 abgebaut werden müssen ${ }^{59}$ ) und die hohen Infrastrukturkosten den Markteintritt neuer Wettbewerber; die bereits angesprochenen Defizite im Telekommunikationssektor treten hinzu.

Schließlich stellte die Reform der Beihilfepolitik ein wesentliches Anliegen der Lissabon-Strategie dar. Hier kam es bei der Verfolgung einiger materielle Zielvorgaben, insbesondere im Bereich der KMU-Förderung, ${ }^{60} \mathrm{zu}$ flankierenden Anpassungsleistungen. So wurde das unionsweite System der Beihilfekontrollen grundlegend reformiert: Die Kommission weist den einzelnen mitgliedstaatlichen Beihilfemaßnahmen im Rahmen regelmäßiger Überprüfungen nun eines von drei „Kontroll-Niveaus“ zu, das die wettbewerbsverzerrenden Auswirkungen der jeweiligen Regelungen widerspiegeln soll. ${ }^{61}$ Indessen sanken die staatlichen

55 Eurostat: Marktanteil des größten Erzeugers im Strommarkt in Prozent des Gesamtmarktes, 15.10.2010.

56 Tilford, S./Whyte, Ph., a.a.O., 36; IW Köln: EU-Liberalisierungspolitik - Erfolge überwiegen. Köln, 2009.

57 Tilford, S./Whyte, Ph., a.a.O., 37.

58 Tilford, S./Whyte, Ph., a.a.O., 35.

59 Europäisches Parlament/Rat: Richtlinie 2008/6/EG.

60 Europäische Kommission: $\operatorname{KOM}(2005) 107$.

61 dies.: $\mathrm{KOM}(2009) 661,14 \mathrm{f}$. 
Beihilfen im Untersuchungszeitraum von $0,67 \%$ (2002) auf $0,53 \%$ des BIP $(2007)^{62}$ - bevor sie im Zuge der Finanz- und Wirtschaftskrise 2008 wieder sprunghaft auf 2,24\% anstiegen. Positiv anzumerken bleibt, dass sich die Vergabe staatlicher Subventionen heute verstärkt auf die Förderung von KMU sowie die Finanzierung von FuE-Maßnahmen konzentriert. ${ }^{63}$

\section{e) Integration der Finanzmärkte}

Der Binnenmarkt für Finanzdienstleistungen wurde erfolgreich ausgebaut, ein im Jahr 1999 verabschiedeter „Aktionsplan für Finanzdienstleistungen“ konnte im Rahmen der Lissabon-Strategie fast vollständig umgesetzt werden. ${ }^{64}$ Als besonders erfolgreich erwies sich die Schaffung des einheitlichen Euro-Zahlungsverkehrsraums SEPA und der Interbank-Überweisungssysteme TARGET bzw. TARGET2. Allerdings offenbarte die Finanzkrise der Jahre 2008 und 2009 massive Defizite in den mitgliedstaatlichen und unionsweiten Finanzmarktaufsichtsystemen, sodass im vergangenen Jahr die Einrichtung eines Europäischen Ausschusses für Systemrisiken und dreier weiterer EU-Behörden beschlossen wurde: der Europäischen Bankaufsichtsbehörde (EBA), der Europäischen Wertpapierbehörde (ESMA) und der Europäischen Aufsichtsbehörde für das Versicherungswesen und die betriebliche Altersvorsorge (EIOPA). ${ }^{65}$ Allerdings gilt die Tragweite der diesen Einrichtungen übertragenen Befugnisse bislang - und unter der Annahme, dass eine europaweite Regulierung tatsächlich wünschenswert ist - als unzureichend; auch fehlt es ihnen an direkt wirksamen und unanfechtbaren Instrumenten zur Regulierung bzw. Steuerung im Krisenfall. So bleibt bereits die bloße Feststellung einer Krisensituation weiterhin dem Rat überlassen; Anweisungen der neu geschaffenen Einrichtungen an Unternehmen und Finanzinstitute sind nur über die mitgliedstaatlichen Finanzmarktaufsichtsbehörden möglich und entfalten keine unmittelbar verbindliche Wirkung. Trotz der krisenbedingt veränderten Wahrnehmung seitens der Mitgliedstaaten gelang es der Kommission und dem Europäischen Parlament im Ergebnis also nur in Teilen, die Finanzmarktaufsicht als Gemeinschaftsaufgabe neu zu definieren.

62 Eurostat: Gesamt Staatsbeihilfen nach Art der Beihilfe in Prozent des BIP, 15.10.2010.

63 Tilford, S./Whyte, Ph., a.a.O., 59.

64 dies., $42 \mathrm{f}$.

65 Europäisches Parlament: Entschließungen vom 22. September 2010 zu Europäische Kommission: KOM(2009)0499, KOM(2009)0501, KOM(2009)0503, KOM(2009)0576, KOM(2009)0502. 
Jenseits der materiellen Diskussion um das Für und Wider einer unionsweiten Regulierung der Finanzmärkte bleibt festzustellen, dass die erreichten institutionellen und verfahrensbezogenen Anpassungen nicht zwangsläufig zu mehr Transparenz und Kontrolle geführt haben; eine sich im Rahmen nachfolgender Krisensituationen ggf. verzögernd auswirkende zeit- wie ressourcenintensive Diskussion um die konkrete Verteilung von Zuständigkeiten und die Verbindlichkeit unionsweiter Regulierungsmaßnahmen erscheint daher unausweichlich.

\section{f) Haushalts- und Wirtschaftspolitik}

Die Bemühungen um eine Konsolidierung der Staatshaushalte erwiesen sich im Zeitablauf als wenig erfolgreich. Zwar sank die gesamteuropäische Schuldenstandsquote geringfügig von 61,9\% (2000) auf 58,8\% des BIP (2007), doch stieg sie im Zuge der Finanz- und Wirtschaftskrise wieder sprunghaft auf 73,6\% $(2009)^{66}$ an. Auch der Blick auf die jährliche Neuverschuldung zeigt, dass von einer unionsweit nachhaltigen Konsolidierung kaum die Rede sein kann: Noch im Wachstumsjahr 2007 lag der durchschnittliche Saldo der staatlichen Gesamthaushalte mit $-0,8 \%$ des BIP deutlich im negativen Bereich. ${ }^{67}$

Die Abstimmung von Finanz-, Wirtschafts- und Währungspolitiken auf EUEbene erwies sich im Verlauf der Krise - trotz einiger protektionistischer Tendenzen in den Mitgliedstaaten - im Ansatz als durchaus reagibel. ${ }^{68}$ Gleichwohl sollte dies nicht darüber hinwegtäuschen, dass die erkennbaren Divergenzen im Rahmen der mitgliedstaatlichen Finanz- und Wirtschaftspolitiken - insbesondere im Euroraum - außerordentlich destabilisierend wirkten. Das Aufweichen des Stabilitäts- und Wachstumspakts durch die , großen“ Mitgliedstaaten Deutschland und Frankreich sowie die unverändert bestehenden Anreize zur relativen Überschuldung angesichts unglaubwürdiger no-bail-out-Bestimmungen trugen maßgeblich zur Staatsschuldenkrise des Jahres 2010 bei, deren Bekämpfung de facto eine Entwicklung der EU hin zu einer Transferunion einleitete. Zwar lassen die jüngst diskutierten Ansätze zur Verstetigung der zunächst provisorischen ,Schutzmechanismen“ eine gewisse Vorsicht der beteiligten Akteure erkennen, doch bleibt weiterhin unklar, ob diese Entwicklung ihr logisches Ende nicht ggf. doch in der Emission gemeinsamer Anleihen (vulgo: Eurobonds) findet und wie

66 Eurostat: Öffentlicher Schuldenstand - in Prozent des BIP, 15.10.2010.

67 ders.: Öffentlicher Finanzierungssaldo - in Prozent des BIP, 15.10.2010.

68 vgl. Fehrmann, T.: The European Union in 2009: a Review, in: ZSE 1/8 (2010), 129-152, hier: 130. 
die geforderte, in Teilen bereits beschlossene Verbesserung der wirtschaftspolitischen Koordination tatsächlich vollzogen werden soll.

Im Ergebnis wurden die „Lissabonziele“ zur Verbesserung der makroökonomischen Koordination und der Rückführung des Schuldenstands maßgeblich von der Finanz-, Wirtschafts- und Staatsschuldenkrise überschattet. Die im Rahmen der Krisenbewältigung ergriffenen Maßnahmen erwiesen sich als wesentlich umfang- und folgenreicher als sämtliche ursächlich auf die „Lissabon-Strategie“ bezogenen Initiativen. Die Krisensituation verwies zudem auf eine Reihe elementarer Defizite in der Koordination der mitgliedstaatlichen Wirtschaftspolitiken und vereitelte zunächst sämtliche Bemühungen zur Konsolidierung der staatlichen Haushalte. Mittelfristig gewannen zwar beide Zielvorgaben an politischer Bedeutung, doch führt gewachsene Aufmerksamkeit nicht zwingend zu entsprechend zielführenden Maßnahmen.

\section{Sozialer Zusammenhalt}

\section{a) Bildung und Ausbildung}

In den Bereichen Bildung und Ausbildung konnten kaum Fortschritte erzielt werden, obwohl sie zu den erklärten Schwerpunkten europäischer Politik im Rahmen der Lissabon-Strategie zählten. Der im Jahr 2005 beschlossene „Europäische Pakt für die Jugend“ und weitere Maßnahmen (wie etwa das Programm „Jugend in Aktion 2007-2013“) fassten zahlreiche der mit Blick auf die „Lissabonziele" definierten Maßnahmen und Leitlinien zusammen; v.a. Projekte von Nichtregierungsorganisationen wurden finanziell gefördert. Von einer maßgeblichen Unterstützung durch die Mitgliedstaaten kann allerdings nicht wirklich die Rede sein. Als ausschlaggebend für die einzige unionsweit konzertierte Reform erwies sich der Bologna-Prozess, ${ }^{69}$ der jedoch eher auf strukturelle Vorgaben und organisatorisch-institutionelle Kontexte als auf materielle Angebote im Universitätsbereich zielte.

Der Anteil der Jugendlichen mit einem Abschluss der Sekundarstufe II wuchs nur leicht von 76,6\% (2000) auf 78,6\% (2009); ${ }^{70}$ die Schulabbrecherquote sank ebenfalls nur geringfügig von 17,0\% (2002) auf 14,4\% (2009) und verweist ischen Bildungsminister. Bologna, 19. Juni 1999.

70 Eurostat: Bildungsstand der Jugendlichen - Prozentsatz der Bevölkerung im Alter von 20-24 Jahren mit mindestens Sekundarstufe-II-Abschluss, 15.10.2010. 
damit weiterhin auf inakzeptable Problemlagen. ${ }^{71}$ Die Bildungsbeteiligung der 18jährigen stieg dagegen etwas deutlicher von 71,2\% (2000) auf 77,0\% (2008) an. ${ }^{72}$ Insgesamt nahm die Zahl der Abgänger mit sekundären und tertiären Bildungsabschlüssen zu; ebenso stieg die Zahl der Studierenden von 15,9 Mio. (2000) auf 19,0 Mio. (2008). ${ }^{73}$ Allerdings fällt die Qualität der Bildungseinrichtungen je nach Mitgliedstaat nach wie vor höchst unterschiedlich aus; die PISAund IGLU-Studien verdeutlichen die sich damit verbindenden Herausforderungen. Auch kam es zu einem nur unwesentlichen Anstieg der öffentlichen Bildungsausgaben als Anteil am BIP. ${ }^{74}$

\section{b) Aktive Beschäftigungspolitik}

Auch im Bereich der Beschäftigungspolitik konnten nur wenige Erfolge verzeichnet werden. Eine EU-weite Datenbank für offene Stellen und Lehrangebote wurde zwar mit dem bereits benannten EURES-System geschaffen, doch blieb deren Nutzung weit hinter den Erwartungen zurück. Im Übrigen wurden neue benchmarks für den Bereich „lebenslanges Lernen“ entwickelt; die Beteiligung an Aus- und Weiterbildungsmaßnahmen stieg im Untersuchungszeitraum leicht von 7,2\% (2002) auf 9,4\% (2008) für die EU-27, für die EU-15 auf immerhin $10,8 \%{ }^{75}$ Zielmarken für den Ausbau der Kinderbetreuung wurden festgelegt, ${ }^{76}$ jedoch im Jahr 2009 knapp verfehlt: 28\% (Ziel: 33\%) der unter Dreijährigen und 83\% (Ziel: 90\%) der Kinder zwischen drei Jahren und dem Pflichtschulalter wurden in entsprechenden Einrichtungen betreut. Die Unterschiede zwischen den Mitgliedstaaten erwiesen sich auch hier als beachtlich. ${ }^{77}$

Die drei Zielmarken im Bereich der Erwerbstätigenquoten wurden im EUDurchschnitt knapp verfehlt: Die Gesamterwerbstätigenquote stieg bis 2009 auf $65,9 \%$, wobei nicht zuletzt bei der Erwerbsbeteiligung von Frauen $(58,6 \%)$ und ders.: Ausgaben für Humanressourcen - in Prozent des BIP, 15.10.2010; in absoluten Zahlen stiegen die Ausgaben um ein Sechstel von 476,0 Mrd. Euro KKS (2001) auf 608,9 Mrd. Euro KKS (2007), vgl. ders.: Öffentliche Gesamtsausgaben für Bildung in Millionen EUR KKS, 15.10.2010.

75 ders.: Lebenslanges Lernen nach Geschlecht, 15.10.2010.

76 Europäischer Rat: Schlussfolgerungen des Vorsitzes, Barcelona, 15. und 16.03.2002.

77 Diese Daten beziehen sich auf die EU-27; Eurostat: Formale Kinderbetreuung nach Altersklassen und zeitlicher Nutzung, 10.12.2010. 
älteren Arbeitnehmern (46,0\%) deutliche Verbesserungen erkennbar wurden. ${ }^{78}$ Verengt man die Berechnungsgrundlage auf die Altersgruppe der 20-64jährigen, so wurden die ersten beiden Ziele mit 70,5\% und 63,0\% (2008) sogar erreicht. Sämtliche Werte liegen jedoch immer noch weit unter denen Japans und der USA (vgl. Abb. 3).

Abbildung 3: Erwerbstätigenquoten im Vergleich (2000- 2009)

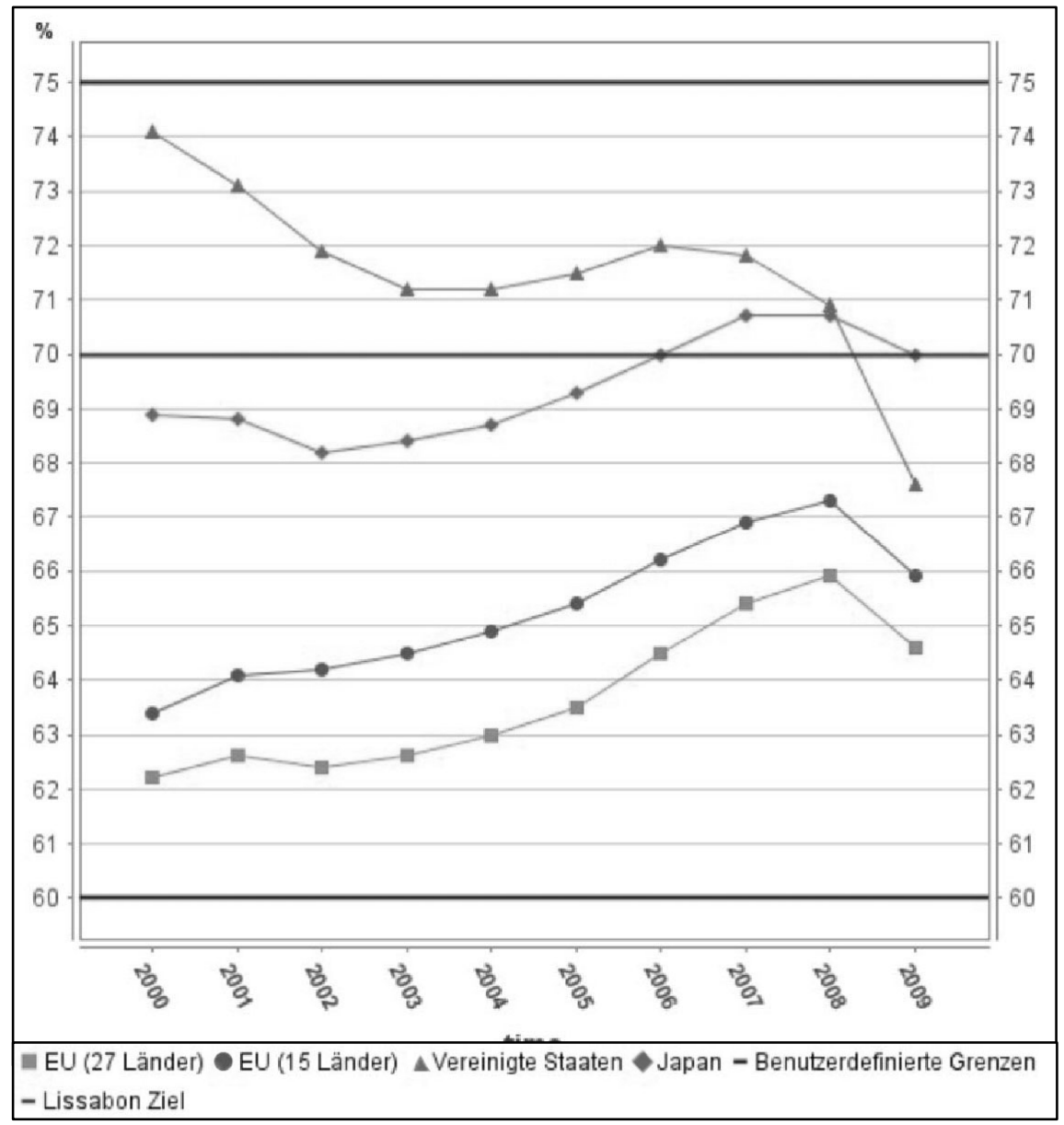

Quelle: Eurostat: Erwerbstätigenquote, 15.10.2010.

78 Eurostat: Erwerbstätigenquote älterer Erwerbstätiger, 15.10.2010; ders.: Erwerbstätigenquote nach Geschlecht, 15.10.2010. 
Die Jugendarbeitslosigkeit sank hingegen nur leicht von 17,4\% (2000) auf $15,5 \%$ (2008). Im Zuge der Krise nahm sie erneut zu und erreichte einen Höhepunkt von $19,7 \%$ im Jahr 2009. ${ }^{79}$

Die Belastung des Faktors Arbeit mit Steuern und Abgaben schließlich wurde kaum reduziert, ${ }^{80}$ die relative Steuerlast für Niedriglohnempfänger fiel nur ansatzweise von $41,0 \%$ (2000) auf 39,9\% (2008), ${ }^{81}$ womit das selbstgesteckte Ziel deutlich verfehlt wurde.

\section{c) Modernisierung der sozialen Sicherungssysteme}

Die geplante unionsweite Umgestaltung der sozialen Sicherungssysteme ist nicht erfolgt. Auf die drängenden demographischen und binnenmarktrelevanten Herausforderungen fand man nur in einzelnen Mitgliedstaaten und auch hier lediglich punktuelle Antworten. Die Reformen bestanden hauptsächlich in der Heraufsetzung des Renteneintrittsalters; es stieg im Unionsdurchschnitt von 59,5 (2001) auf 61,4 Jahre (2008). ${ }^{82}$ Ein EU-weiter Ansatz war zwar formal vorgesehen, wurde aber nicht verwirklicht. Die zum Thema „Sozialschutz“ eingerichtete high-level group beschränkte sich in ihrem Aufgabenfeld auf lediglich beratende Funktionen. ${ }^{83}$ Wie geplant, wurde im Übrigen eine Studie zur Tragfähigkeit der Altersversorgungssysteme in Auftrag gegeben. ${ }^{84}$ Sie identifiziert - wenig überraschend - die Alterung der Gesellschaft, das Sinken der Geburtenraten und die Integration von Einwanderern als zentrale Herausforderungen. Es fehlt mithin eine gemeinsame Herangehensweise an die Modernisierung aller sozialen Sicherungssysteme; die OMK erbrachte hierzu so gut wie keine Ergebnisse.

\section{d) Soziale Integration}

Das Ziel der Förderung sozialer Integration wurde in zahlreichen Aufgaben- und Politikbereichen aufgegriffen. Als erfolgreich erwiesen sich v.a. EU-Initiativen zur Gleichstellung von Männern und Frauen sowie zur Förderung tatsächlicher Chancengleichheit. Besonders durch den Vertrag von Lissabon wurde das Tätigmereinkommen zum Arbeitnehmerentgeld insgesamt, 15.10.2010.

81 ders.: Steuerlast auf Arbeitskosten, 15.10.2010.

82 ders.: Durchschnittliches Erwerbsaustrittsalter, 15.10.2010.

83 Rat: 2004/689/EG.

84 Europäische Kommission: $\operatorname{KOM}(2000) 622$. 
keitsfeld „Soziales“ auf EU-Ebene stark ausgebaut. ${ }^{85}$ In den Mitgliedstaaten hingegen fielen die Bemühungen dagegen erneut sehr unterschiedlich aus. Da v.a. im Bildungsbereich und beim Umbau der sozialen Sicherungssysteme kaum Fortschritte gemacht wurden, konnte auch die soziale Integration nur unwesentlich vorangetrieben werden. Die „Armutsgefährdungsquote" verzeichnete im Jahr 2009 mit 16,4\% der Gesamtbevölkerung einen weiterhin sehr hohen Wert. ${ }^{86}$

\section{e) Umweltpolitik}

Die Umweltziele wurden nur zum Teil erreicht. Der Anteil Erneuerbarer Energien an der Stromerzeugung lag 2007 bei 15,6\%; ${ }^{87}$ die Berechnungen der Kommission gehen davon aus, dass das Ziel von 21\% auch bis 2010 nicht erreicht wird. $^{88}$ Der Anteil der Kraft-Wärme-Kopplung stieg auf nur $11 \%$ (2008) der Brutto-Gesamtelektrizitätserzeugung, ${ }^{89}$ das Ziel von $18 \%$ wurde mithin grob verfehlt. Dagegen konnten die EU-27 ihre Treibhausgasemissionen bis zum Jahr 2007 auf 90,7\% des Vergleichswerts von 1990 reduzieren. ${ }^{90}$ Damit wurde das Ziel von $92 \%$ erreicht, auch wenn diese Entwicklung vor allem dem Zusammenbruch der sozialistischen Wirtschaftssysteme in den ehemaligen Ostblockstaaten $\mathrm{zu}$ verdanken sein dürfte. Insgesamt wuchs die Bedeutung der Themenfelder Umweltschutz und Energieeffizienz deutlich und führte zunehmend zu Forderungen nach unionsweit koordinierten Maßnahmen. Im Ergebnis vertritt die EU gegenüber Drittstaaten, etwa im Rahmen der UN-Klimagipfel, inzwischen häufig eine einheitliche umweltpolitische Verhandlungsposition.

\section{Gesamtindikatoren}

Die Bemühungen um eine „Messung“ der Wettbewerbsfähigkeit werden in der Fachöffentlichkeit wie in der Praxis kontrovers diskutiert. Dies gilt auch für die mit der „Lissabon-Strategie“ vereinbarten Indikatoren.

Siehe dazu detailliert: Rodrigues, M. J.: The Lisbon Agenda and European Governance. Workshop IV: The Lisbon Agenda and European Governance, 29 February 2008, Calouste Gulbenkian Foundation, Technical Report. London: Institute for Strategic and International Studies, 2008.

86 Eurostat: Armutsgefährdungsquote nach Sozialleistungen und Geschlecht, 15.10.2010.

87 Europäische Kommission: EU Energy and Transport in Figures. Statistical Pocketbook, 2010, 41.

88 dies.: $\operatorname{KOM}(2009)$ 192, 3.

89 Eurostat: Kraft-Wärme-Kopplung - Prozent der Brutto-Gesamtelektrizitätserzeugung, 15.10.2010.

90 Europäische Kommission: EU Energy and Transport in Figures, a.a.O., 183. 
Abbildung 4: Arbeitsproduktivität im Vergleich zum EU-27-Durchschnitt (2009)

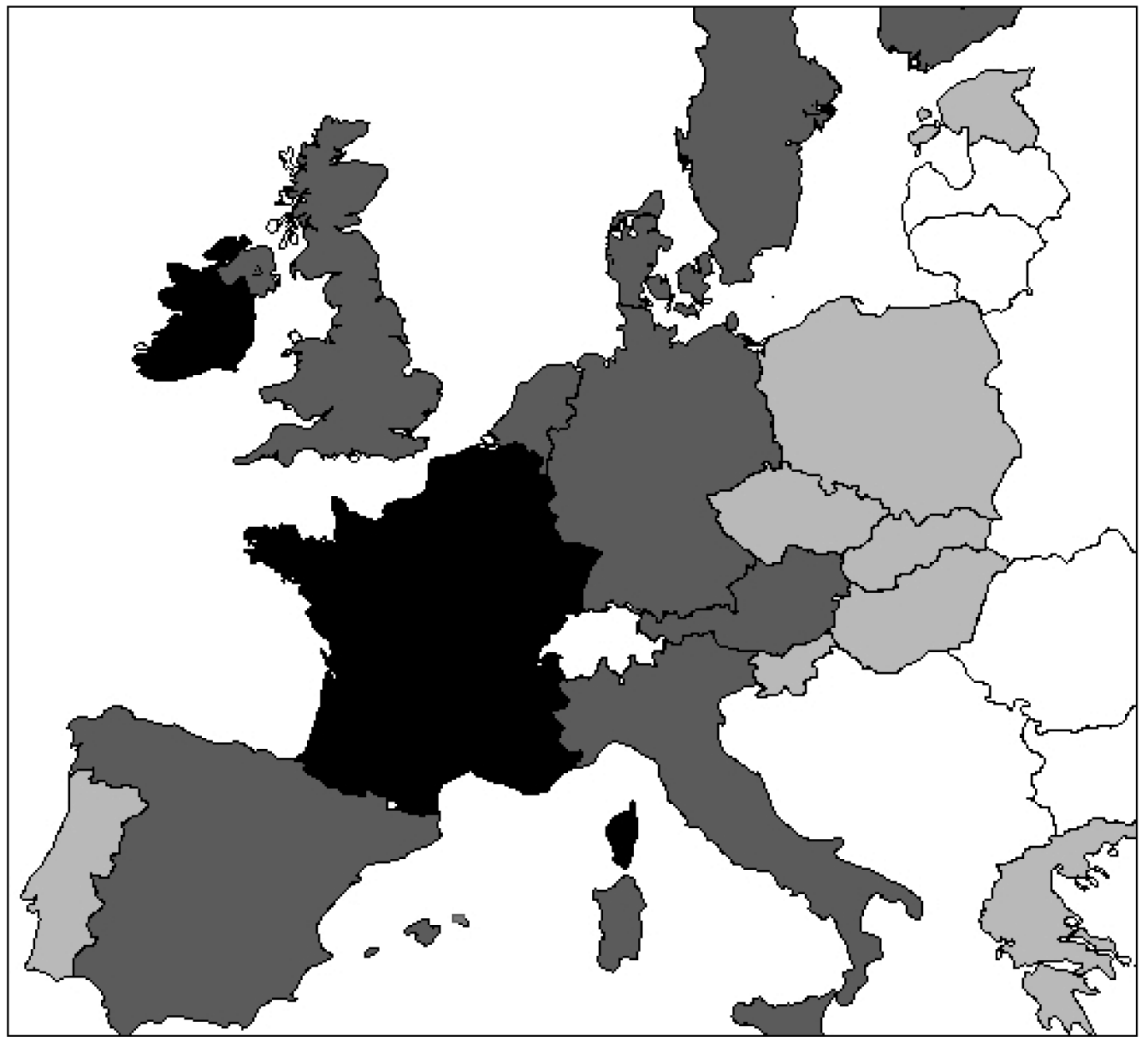

Legende: EU-27 (aus Darstellungsgründen exkl. Zypern und Malta); Durchschnittswert - 100; schwarz - 120 bis 169,8; dunkelgrau - 100 bis 120; hellgrau - 60 bis 100; weiß - 38,5 bis 60; Quelle: Eurostat: Arbeitsproduktivität je Beschäftigten - BIP in KKS je Beschäftigten, 15.10.2010.

Mit Blick auf die Arbeitsproduktivität finden sich noch immer massive Unterschiede zwischen den Mitgliedstaaten (vgl. Abb. 4). ${ }^{91}$ Wenig verwunderlich ist zudem, dass sich die erwünschte Steigerung des EU-Durchschnittswerts auf das Niveau der international führenden Staaten nicht erreicht wurde: In den USA beschäftigte Arbeitskräfte waren im Jahr 2008 weiterhin deutlich produktiver als ihre europäischen Kollegen $(+37,8 \%)$. Allerdings ist die Aussagekraft dieses Indikators grundsätzlich kritisch zu beurteilen: So wird hier vornehmlich auf den Quotienten aus BIP und Beschäftigtenzahl abgestellt, unterschiedliche Regelungen zu Arbeitszeiten, Urlauben und Überstunden jedoch nicht berücksichtigt.

91 ders.: Arbeitsproduktivität je Beschäftigten - im Vergleich zu EU-27 (EU-27 = 100), 15.10.2010. 
Aus Sicht der Vergleichbarkeit ist daher die Zahl der tatsächlich geleisteten Arbeitsstunden als Berechnungsgrundlage wesentlich zielführender, doch fehlen hierfür häufig verlässliche Daten. Schließlich geht die Produktivitätsmessung von der Annahme aus, dass sich Qualitätsdifferentiale zwischen den hergestellten Produkten bzw. den erbrachten Dienstleistungen vollständig im Preis niederschlagen. Angesichts durchaus bedeutsamer Informationsasymmetrien sowie weiterhin bestehender Marktrigiditäten darf diese Prämisse allerdings angezweifelt werden, sodass jede Produktivitätsmaßzahl mit entsprechender Vorsicht betrachtet werden sollte.

Die unternehmerische Investitionstätigkeit blieb im Verhältnis zur Wirtschaftsleistung nahezu unverändert und liegt - wie bereits im Jahr 2000 - bei 18,4\% des BIP (2008), bei äußerst divergenter Entwicklung in den einzelnen Mitgliedstaaten. $^{92}$

Abbildung 5: BIP pro Kopf in Kaufkraftstandards (2000-2008)

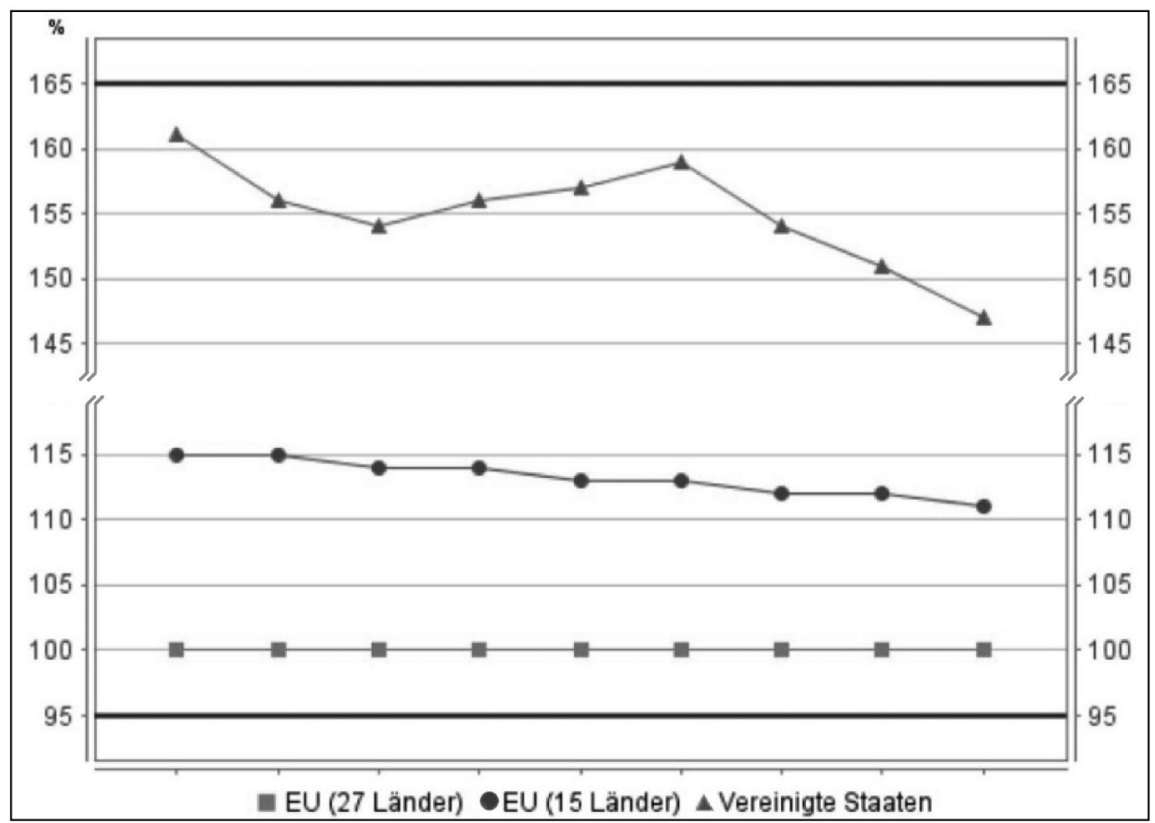

Quelle: Eurostat: BIP pro Kopf in Kaufkraftstandards (KKS) $(\mathrm{EU}-27=100)$, 15.10.2010. 
Schließlich verringerte sich der Abstand zwischen alten und neuen Mitgliedstaaten mit Blick auf die durchschnittliche Wirtschaftsleistung kontinuierlich; im Jahr 2009 betrug der „Vorsprung“ der EU-15 auf die EU-27 nur noch etwas über 10\%. Zwar verringerte sich der Abstand zu den USA, doch konnte das Ziel einer substantiellen Angleichung erkennbar nicht erreicht werden (vgl . Abb. 5). ${ }^{93}$

Mit Blick auf die benannten Aggregate hat konnte die Wettbewerbsfähigkeit des europäischen Wirtschaftsraums also kaum gesteigert werden. Erweitert man die Analyse auf die in der „Lissabon-Strategie“ vorgesehenen Einzelmaßnahmen, so ergibt sich ein lediglich marginal besseres Bild: Auch hier wurden die meisten konkreten Zielsetzungen deutlich verfehlt, obwohl eine Reihe von Indikatoren wie bereits dargestellt - auf grundsätzlich positive Entwicklungstendenzen verweist. Eine ähnliche Bilanz ist mit Blick auf die mitgliedstaatliche Ebene zu ziehen: Zwar sind inzwischen deutliche Angleichungsprozesse erkennbar, doch sehen sich die Nationalstaaten nach wie vor mit in Teilen äußerst heterogenen Ausgangsbedingungen konfrontiert.

\section{Verfahren und Instrumente}

Die im Rahmen der Umsetzung der Strategie eingesetzten Instrumente entwickelten sich zu wesentlichen Bestandteilen der europäischen Politikgestaltung. Vor allem die Einführung der „Integrierten Leitlinien“ und der dreijährigen Planungs- und Evaluationszyklen hat die Arbeit auf der EU-Ebene positiv beeinflusst. ${ }^{94}$ Der mehrjährige Orientierungsrahmen erlaubte eine materielle Fokussierung und reduzierte den Berichtsaufwand, ${ }^{95}$ verhinderte aber gleichzeitig eine vertiefte operative Konkretisierung der entsprechenden Vorhaben. ${ }^{96}$

Mit Blick auf die bei der Union angesiedelten Kompetenzbereiche wurde zwar eine gesamthafte Konzertierung der gemeinschaftlichen Maßnahmen unter den Leitsätzen der „Lissabon-Strategie“ in Angriff genommen und konnte eine Vielzahl von Maßnahmen unter einheitlichen materiellen Vorgaben gebündelt und ggf. reformiert werden, ${ }^{97}$ doch blieb die Kommission in den jährlich veröffentlichten Fortschrittsberichten zunächst den Nachweis schuldig, dass die darin

94 Begg, I.: a.a.O., 22.

95 Europäische Kommission: SEK(2010) 114, 7.

96 Deroose, S./Hodson, D./Kuhlmann, J.: The Broad Economic Policy Guidelines: Before and After the Re-launch of the Lisbon Strategy. in: JCMS 2008 Volume 46, Number 4, 839ff.

97 Begg, I., a.a.O. 
benannten Initiativen tatsächlich problemadäquate Ansätze darstellen - und wurden die selbstbenannten Zielsetzungen schließlich eindeutig verfehlt. Dies ist maßgeblich darauf zurückzuführen, dass die Gemeinschaft in vielen Aufgabenfeldern, auf die sich die „Lissabon-Strategie“ bezieht, nur über eng begrenzte Handlungskompetenzen verfügt und somit auf die Kooperation der Mitgliedstaaten sowie die Koordination der von ihnen verfolgten Maßnahmen angewiesen bleibt. Zwar versuchte die Kommission die jeweiligen Zuständigkeiten in ihren Berichten zu benennen, doch gelang es ihr kaum, entsprechende Vorgaben im Rat konsensfähig zu machen. ${ }^{98}$ Während sie in den ersten Jahren im Rahmen von „Länderspezifischen Empfehlungen“ bzw. „Durchführungsberichten“ deutlich auf spezifische Umsetzungsschwierigkeiten einging, bevorzugte sie im weiteren Verlauf eher allgemeine Formulierungen. ${ }^{99}$ Für eine regelmäßige und präzise Überprüfung der nationalstaatlichen Maßnahmen verfügt die Kommission offenbar weder über entsprechende empirisch-analytische Zugänge noch über die notwendige politische Unabhängigkeit.

Mit Blick auf die mitgliedstaatlichen Zuständigkeitsbereiche erwies sich die OMK als wenig erfolgreiches Instrument zur Koordination europäischer wie nationalstaatlicher Politiken. Zwar einigte man sich mit der Formulierung gemeinsamer benchmarks auf eine Verfahrensform, doch wurde der ursprünglich vorgesehene intensive Erfahrungsaustausch in nur äußerst begrenztem Umfang realisiert. Meist verzichtete man auf eingehendere Analysen der jeweiligen Ausgangssituation sowie der damit verbundenen Entwicklungspotentiale in den Mitgliedstaaten und konnte sich deshalb nur selten auf konkrete Vollzugsschritte und Berichtspflichten einigen. Beschlossene Maßnahmen blieben somit im Ungefähren; Absichtserklärungen und die Formulierung abstrakter Zielwerte dominierten das Tagesgeschäft. Das erkennbar intendierte naming and shaming bleibt auch weiterhin solange funktionslos, als sich damit keine Sanktionsmechanismen verbinden: ${ }^{100}$ „soft law brings soft results“. ${ }^{101}$ Im Ergebnis dürfte sich die OMK nur dann als entwicklungsfähig erweisen, wenn man künftig vom Postulat der Einbindung aller Mitgliedstaaten in jede Koordinierungsmaßnahme Abstand nimmt. Die freiwillige Zusammenführung von Mitgliedstaaten mit ähnlichen

98 Collignon, St:: The Lisbon Strategy, Macroeconomic Stability and the Dilemma of Governance with Governments. Harvard University/London School of Economics, 2006, 11.

99 Wyplosz, Ch.: The Failure of the Lisbon Strategy. VOX, 2010, 3.

100 Collignon, St., a.a.O., 11.

101 Zgajewski, T./Hajjar, K.: The Lisbon Strategy: Which Failure? Whose Failure? And Why? Egmont Paper No. 6, 2005, 23. 
Problemlagen würde einen intensiveren Austausch, realistischere benchmarks und ehrgeizigere Vollzugsanstrengungen ermöglichen. Politische Ängste vor einem ,Europa der zwei Geschwindigkeiten' dürften ein entsprechendes Vorgehen allerdings verhindern - unabhängig von der mit Blick auf die Gemeinschaftswährung und den Schengen-Besitzstand bereits seit Längerem erkennbaren Faktizität einer solchen ,Entkopplung'.

Schließlich unterlag die Auswahl der einzelnen Zielwerte in Teilen deutlicher Kritik: ${ }^{102}$ Die über 100 Einzelindikatoren erwiesen sich oft als nicht ausreichend aussagekräftig und in Teilen als ungleichgewichtig. Hinzu trat, dass Eurostat in einigen Fällen nur unzureichendes Datenmaterial vorlegte. ${ }^{103}$

\section{Gesamtbewertung}

Im Ergebnis gilt die „Lissabon-Strategie“ in Wissenschaft und Praxis als weitgehend gescheitert. ${ }^{104}$ Zwar konnten in einigen Bereichen beträchtliche Fortschritte erzielt werden und kam es zu einer gewissen Aufmerksamkeitssteigerung für die in der Strategie benannten Themenfelder, doch verband sich dies meist weniger mit einer Umsetzung der „Lissabonziele“ als mit eher selbstverständlichen, durch die technologische bzw. wirtschaftliche Entwicklung induzierten und unabhängig von der europäischen Politik erbrachten Anpassungsleistungen in den Mitgliedstaaten.

Die in Teilen beträchtliche Heterogenität der Ausgangslagen und Entwicklungspotentiale stellte die Formulierung einheitlicher Zielwerte ab ovo in Frage; als wenig verwunderlich kann gelten, dass Mitgliedstaaten mit ungünstiger Ausgangsposition in den meisten Fällen auch eine vergleichsweise ungünstige Entwicklung zeitigten. ${ }^{105}$ In den wohlhabenderen Mitgliedstaaten wurden hingegen entsprechende Reformvorhaben v.a. aus innenpolitischen Erwägungen heraus verfolgt, die „Lissabonziele“ spielten - wenn überhaupt - nur eine untergeordne-

102 Siehe hierzu bspw. OECD: Revising the Lisbon Strategy through OECD Innovation Strategy, 2009.

103 Heinemann, Fr./Hauptmeier, S./Kalb, A./Kappler, M.: "LiMa-Benchmark": Eine Performance-Analyse der EU-Mitgliedstaaten im Licht der Lissabon- und Maastricht-Zielsysteme. Bertelsmann Stiftung, 2007, 6.

104 Vgl. u.a. die Äußerungen des schwedischen Ratsvorsitzes im Jahr 2009: "Even if progress has been made, it must be said that the Lisbon Agenda, with only a year remaining before it is to be evaluated, has been a failure.", in: Reinfeld, F./Borg, A., Zeitungsinterview, Dagens Nyheter, 2009; zitiert von: http://www.euractiv.com/en/priorities/sweden-admits-lisbon-agenda-failure/article-182797.

105 Tilford, S./Whyte, Philip, a.a.O., 70. 
te Rolle. ${ }^{106}$ Zwar gelang der Kommission in ihrem Kompetenzbereich eine beachtliche inhaltliche Bündelung von Einzelmaßnahmen, ${ }^{107}$ doch konnte auch dies dem grundsätzlichen Dilemma sehr ambitionierter Zielvorgaben bei gleichzeitig nur unzureichender operativer Grundausrichtung und heterogenen Kompetenzstrukturen nicht überzeugend entgegenwirken.

Als Grund für das Verfehlen vieler benchmarks wird zwar v.a. auf einen vermeintlichen Mangel an ,politischem Willen` seitens den Mitgliedstaaten verwiesen, doch fällt mit Blick auf die Anreizsysteme der Lissabon-Instrumente ein anderer Faktor deutlich stärker ins Gewicht: das Fortbestehen allzu bekannter collective action problems. ${ }^{108}$ So betrachtet die Strategie ausschließlich potentielle spill-over-Effekte als Motivation und Anreiz, ohne auch politische und wirtschaftliche Kosten mit zu bedenken. ${ }^{109}$ Zudem wurden mit einigen Indikatoren Kennzahlen festgelegt, die durch staatliches Handeln kaum direkt beeinflusst werden können. Schließlich spielte die - besonders für den Euroraum - bedeutsame Koordinierung makroökonomischer Wirtschaftspolitiken eine nur untergeordnete Rolle im Rahmen der Strategie, ${ }^{110}$ eine Auslassung, die sich spätestens mit Beginn der Finanz- und Wirtschaftskrise, rächte‘. Im Ergebnis wurden die eigentlichen Anknüpfungspunkte für systemische Reformen häufig schlicht übergangen.

Die meisten der zahlreichen Einzelziele erwiesen sich im vorgegebenen Zeitraum somit als „unerreichbar“, was bei den beteiligten Akteuren zu einem erheblichen Verlust an Glaubwürdigkeit führte ${ }^{111}$ und die „Lissabon-Strategie“ in Teilen gar kontraproduktiv erscheinen ließ. Es wurde erkennbar, dass man Ziele zwar vergleichsweise eindeutig zu formulieren vermochte, den Weg zu ihrer Erreichung jedoch in vielen Fällen vernachlässigte; ${ }^{112}$ spezifische Kontextbedingungen und die notwendige Vollzugsorientierung wurden allzu häufig ausgeblendet.

106 dies., 3.

107 Zgajewski, T./Hajjar, K., a.a.O., 18.

108 Collignon, St., a.a.O., 7.

109 Zgajewski, T./Hajjar, K., a.a.O., 18.

110 Collignon, St., a.a.O., $22 \mathrm{ff}$.

111 Ebd., 1

112 Begg, I.: The Lisbon Strategy Post 2010, in: Bundesministerium für Wirtschaft und Arbeit Österreich (Hg.): Die Zukunft der Wirtschaftspolitik der EU. Beiträge zum Diskussionsprozess „Lissabon Post 2010“, Wien, 2008, 12. 


\section{Fazit und Ausblick}

Bis zur Veröffentlichung ihres Nachfolgers „Europa 2020“ stellte die „LissabonStrategie" das umfassendste und ambitionierteste langfristige Planwerk der EU dar. Sie gilt zurecht als gescheitert, da die (horizontal wie vertikal) beteiligten Akteure die ehrgeizigen und nicht selten, vollmundig' verkündeten Zielsetzungen nicht zu erfüllen vermochten. Obwohl Aufwand und Ergebnisse in keinem wirklich akzeptablen Verhältnis stehen, bewirkte die Strategie einige wenige Fortschritte in der Sache wie im Verfahren.

In der Sache wurde deutlich, dass die Verkündung politischer Ziele noch nicht deren Vollzug bedeutet und es diverser auch operativer Anstrengungen bedarf, um ,europäische Politik ' ergebnisorientiert zu gestalten und umzusetzen. ${ }^{113}$ Die in vielen Fällen dennoch erkennbaren positiven Entwicklungen verweisen auf eine vergleichsweise günstige Ausgangslage, sodass eine kluge, realistische und besonnen umgesetzte gemeinsame Strategie durchaus auf fruchtbaren Boden fallen könnte.

Im Verfahren gelang es, den Kommunikationsprozess zwischen den Akteuren zu verbessern und Lernprozesse auszulösen, die allerdings bislang ohne nachhaltige Konsequenzen blieben. Vor allem die OMK bedarf einer dringenden Überprüfung, um mitgliedstaatliche Maßnahmen künftig zielgerichteter koordinieren und der Union nötigenfalls auch in gemeinschaftsfremden Zuständigkeitsbereichen ein effektiveres Handeln ermöglichen zu können.

Für die Kommission schließlich entwickelte sich die Strategie trotz aller Rückschläge zu einem wichtigen Instrument ihrer politischen Arbeit. Materielle Defizite und bestehende Legitimationsschwächen konnten überdeckt und die positive öffentliche Wahrnehmung von Teilen des Programms genutzt werden, um die Arbeit der Organe der EU im Allgemeinen und die der Kommission im Besonderen verstärkt im Bewusstsein der ,Völker Europas` zu verankern. Mit der Strategie suchte sie die Mitgliedstaaten politisch enger an sich zu binden und sich selbst eine koordinierende Rolle zu übertragen, der sie allerdings nicht immer gewachsen war. Zwar trat sie als agenda setter und ,Triebkraft' auf und reizte dabei den Rahmen des Subsidiaritätsprinzips bis an seine Grenzen hin aus, doch wurde auch deutlich, dass gerade an den Schnittstellen zwischen den Gemeinschaftsaufgaben und den Zuständigkeitsbereichen der Mitgliedstaaten das

113 Vgl. hierzu grundlegend Hesse, J.J./Grotz, F.: Europa professionalisieren. Kompetenzordnung und institutionelle Reform im Rahmen der Europäischen Union, Berlin, 2005. 
Bewusstsein für operative Fragen und den koordinierten Vollzug politischer Maßnahmen nur unzureichend ausgeprägt war. Somit wandelte sich das Verhältnis zwischen der Kommission und den Mitgliedstaaten im Ergebnis zugunsten eines pragmatischeren Verständnisses europäischer Politik, das eine weitere ,Europäisierung' von Themenfeldern und Aufgabenbereichen nur dann als notwendig und politisch konsensfähig erscheinen lässt, wenn deren nachfolgende Vorteile zweifelsfrei dokumentiert werden können. Ob die neue „Wachstumsstrategie Europa 2020“ die angesprochenen Defizite auszugleichen und die entsprechenden Nachweise zu erbringen vermag, muss einer gesonderten Betrachtung überlassen bleiben.

von Sarah Ciaglia, Thomas Fehrmann und der ZSE-Redaktion 